Elskamp, F.; Kruggel-Emden, $\mathrm{H}$.

\title{
DEM simulations of screening processes under the influence of moisture
}

Journal article | Accepted manuscript (Postprint)

This version is available at https://doi.org/10.14279/depositonce-8317

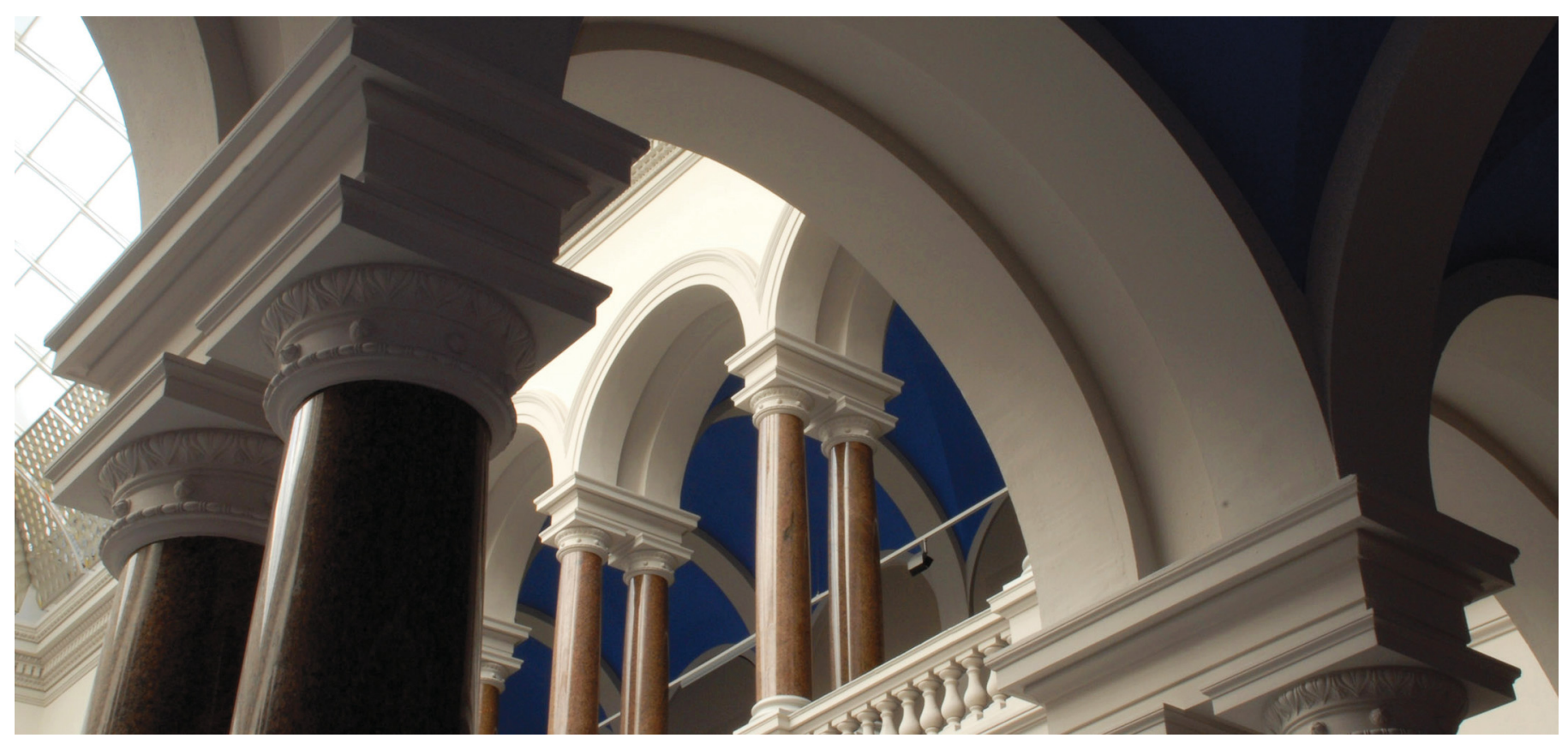

Elskamp, F., \& Kruggel-Emden, H. (2018). DEM simulations of screening processes under the influence of moisture. Chemical Engineering Research and Design, 136, 593-609.

https://doi.org/10.1016/j.cherd.2018.06.022 


\section{DEM simulations of screening processes under the 2 influence of moisture}

3 Frederik Elskamp ${ }^{1 *}$, Harald Kruggel-Emden ${ }^{1}$

$4{ }^{1}$ Mechanical Process Engineering and Solids Processing, Technische Universität Berlin, Ernst-

5 Reuter-Platz 1, D-10587 Berlin, Germany

6 *Corresponding author. Tel.: +49-30-314-23496; Fax: +49-30-314-26432

7 E-mail address: frederik.elskamp@tu-berlin.de

\section{Abstract}

9 In a wide field of applications, screening is required to separate bulk materials according to their particle sizes. Due to environmental, material or process related effects, particles frequently prevail in moist conditions, which is not preferred due to attractive forces altering the screening efficiency, but often not preventable. As for the design of dry screening processes detailed particle-based simulation approaches like the discrete element method (DEM) and phenomenological models are available, a step towards meeting the requirements for real particle systems under moist conditions is made. Therefore, batch screening under the influence of moisture is investigated experimentally and by using DEM simulations involving different sized polyoxymethylene and glass spheres. For this purpose, a DEM code is extended to calculate forces caused by liquid bridges, forming out between particles or walls close to each other under moist conditions. Thereby, the bridge formation and rupture and the liquid distribution are considered. First, the DEM framework is validated against experiments by monitoring the capillary and viscous force acting on two liquid bridge contact partners. Further extensive validations are performed by comparing the fraction retained over time and the final liquid distribution for discontinuous screening under the influence of various amounts of liquid for different mechanical agitations in experiments and simulations. Finally, the detailed liquid distribution over time in the DEM simulations is examined and general conclusions are drawn. The overall aim is to use the framework and the respective data, to extend phenomenological process models for screening under moist conditions in subsequent studies.

Keywords: Discrete element method (DEM); Capillary and viscous forces; Liquid bridge; Screening; Moisture

\section{Introduction}

31 In order to separate bulk material in many industrial applications, screening is a technical 32 simple but important process step to classify particles according to requested size class 
specifications $[1,2]$. Until now, screening under the influence of a liquid phase has rarely been investigated. Pure experimental investigations have been performed, e.g. by Guerreiro et al. [3], who focused on the optimization of the residual moisture content and the separation efficiency on the screen. Further research addressed the vibrating dewatering of bulk material on screens [4] or the effect of wet screening on particle size distribution [5]. The performance of wet and dry screening was exemplarily compared by Robertson et al. [6].

To avoid extensive experimental tests, the discrete element method (DEM), which was first introduced by Cundall and Strack [7], can be applied to study screening and its sub-processes in detail. It has been proven as a suitable tool in various investigations on screening [8-12]. However, the particles were assumed as dry or the influence of the fluid was omitted in these studies. In contrast, some researchers concentrated on wet screening applications. In the investigation by Dong and Yu [13], the particle flow and the complex screen geometry as well as a simplified description of the fluid flow modelled by computational fluid dynamics was taken into account. Other researchers coupled discrete element simulations with methods used for simulating the dynamics of continua like the smoothed particle hydrodynamics (SPH) for wet screening. In the work by Fernandez et al. [14], one-way coupled DEM-SPH simulations are performed to take the particle as well as the fluid flow into account. Therein, the particles are completely covered by the surrounding fluid, which reduces the bonding of particles, removes pile-ups on the screen and supports the transport of fine particles through the apertures resulting in an improvement of the screening efficiency. In contrast, a slight natural amount of water in the material can result in bonding of particles and in a lower screening efficiency [5]. Therefore, it is preferable to perform screening either completely under fully dry or wet conditions. Nevertheless, as fully dry or wet conditions cannot always be ensured, the screening behavior under the influence of moisture must be better understood to determine the impact in respective processes. Since discrete element simulations seem to be suitable also for this purpose, the contact forces in the DEM have to be extended by forces, which arise from the presence of liquid. An overview of theoretical developments of discrete particle simulations of dry and wet particulate systems is given by Zhu et al. [15]. Besides systems where the particles are completely surrounded by a liquid, most of the researches are limited on applying a small amount of liquid to ensure only individual capillary bridges without liquid in the pores inbetween the particles.

The forces acting on particles due to the formation of liquid bridges were studied in many investigations without (e.g. [16-20]) and with (e.g. [21-25]) using particle based simulation methods such as the DEM and by applying models for the forces, formation and rupture of the liquid bridges (first method) or by adjusting the restitution coefficient to account for the affected 
contacts (second method). The first method has the advantage of being more realistic when the resulting restitution coefficient is close to zero, due to taking the possible adhering of contact partners into account, which is not possible in the second method. However, resulting lubrication forces have to be considered by additional models, whereas in the second method, these forces are directly considered in addition to the capillary forces [26]. Latter approach was used by several researchers, among them Fu et al. [27] who studied the impact behavior of wet granules on dry surfaces to obtain the contact behavior under such conditions. A lot of effort was put into measuring and investigating the restitution coefficient of various dry particles on different wet surfaces, first experimentally and numerically by Antonyuk et al. [28,29] and later experimentally complemented by Crüger et al. [30,31]. In addition, Sutkar et al. [32], developed expressions for the wet restitution coefficient by energy and dimensional analysis. The interaction between wet particles in a fluidized bed by considering a restitution coefficient which is varied in time and space depending on the moisture content was studied numerically by van Buijtenen et al. [33].

Some of the researchers, who modelled liquid bridges, only considered capillary forces while others also took the influence of viscous forces into account, which are more important for large liquid viscosities or in systems with particles under high velocities. Kralchevsky and Nagayama [34] give an overview and comparison about lateral capillary forces. One of the first expressions for the capillary force of a liquid bridge based on its total energy was given by Israelachvili [20]. Lambert et al. [17] and Gabrieli et al. [35] compared two different capillary force methods, namely the energetic method based on the derivation of the total interfacial energy and a method based on the Young-Laplace equation where the pressure and tension terms obtained from the meniscus profile are summed up. Therein, a further subdivision in gorge (e.g. [36]) and boundary (e.g. [37]) methods can be made. Soulié et al. [38] and Richefeu et al. [39] proposed an approximate exponential fitting of the Young-Laplace equation for unequal sized spheres and offered an equation for calculating the capillary force during and after a direct particle contact. A commonly used model for the capillary forces, based on the pressure difference across the liquid bridge, was later presented by Rabinovich et al. [16]. They proposed and validated equations for liquid bridge contacts between a sphere and a wall as well as between two unequal sized spheres with different wettability. Another capillary liquid bridge model was developed by Willett et al. [18], providing equations for the force acting between two unequal sized spheres and their rupture distance. Weigert and Ripperger [19] introduced a liquid bridge model, where besides the capillary force, the bridge volume is calculated from the half-filling angle. A comparison of the three aforementioned capillary bridge models in terms of their applicability in a DEM framework has been carried out by Gladkyy and Schwarze [40]. Furthermore, Lian et al. [36] developed a closed form equation for capillary 
bridges between spherical particles which was later extended by Lian and Seville [41] to calculate capillary bridges more accurately with general closed-form expressions also applicable for unequal sized spheres, differing wettability and varying liquid bridge volumes.

Adams and Perchard [42] derived a viscous force model in the normal direction, which was implemented by several other authors (e.g. [24,36,43]). Pitois et al. [44] investigated the viscosity effects between two moving spheres connected by a liquid bridge and extended the aforementioned model, which was also applied by Washino et al. [25]. A commonly used liquid bridge viscosity model in tangential direction is the extended model by Goldmann et al. [45], which was applied by many authors (e.g. [25,36,46,47]). In further studies, Pitois et al. [48] proposed a dynamic rupture distance and validated their liquid bridge viscosity model.

The formation, shape, liquid volume and liquid redistribution after rupture of a bridge was intensively studied by Pepin et al. [49] as well as by Shi and McCarthy [43]. Schmelzle and Nirschl [50] studied mixing of dry and wet granular material with the DEM and performed a regression analysis for the liquid bridge force which also gave information about the rupture distances and transfer ratios. All three studies assumed a constant liquid bridge volume between formation and rupture. To overcome this lack, Wu et al. [51] developed a dynamic liquid bridge formation model for equal sized particles, capable of predicting the actual liquid volume in the bridge and on the particles. In particular, this is relevant for highly viscous liquids and short collisions.

Although mostly small liquid amounts are applied to the particles, the liquid bridges between them can become big enough to overlap with other bridges nearby. To prevent this, Scholtès et al. [52] proposed a numerical procedure to identify such overlapping bridges. For the case when a larger amount of liquid is present in a process, Melnikov et al. [53] provided a model to combine capillary bridges, menisci and fully saturated pores to liquid clusters. Additionally, for the case of such a funicular state, Wu et al. [54] investigated the forces and the rupture of liquid bridges between three spherical particles.

One of the first studies of larger particulate systems with equal sized spheres in the DEM was conducted by Yang and Hsiau [55] who applied powders in a 2D vibrated bed under the influence of a small amount of liquid. An early 3D study of a packed bed with wet coarse uniform spheres was performed by Yang et al. [56]. The flow of dense cohesive granular materials in a homogeneous plane shear without interstitial fluid was investigated by Rognon et al. [57] with the help of the molecular dynamics method in 2D. Based on this, the major laws for modelling the flow of wet granular media in the pendular state and the influence of capillary effects were examined applying three dimensional simulations by Khamseh et al. [58]. Among further applications which were simulated and studied with the help of the DEM, Radl et al. [21] 


$$
\begin{aligned}
& m_{i} \frac{d^{2} \vec{x}_{i}}{d t^{2}}=\vec{F}_{i}^{c}+\vec{F}_{i}^{l}+m_{i} \vec{g}, \\
& I_{i} \frac{d \vec{\omega}_{i}}{d t}=\vec{M}_{i}
\end{aligned}
$$

163 with particle mass $m_{i}$, particle acceleration $d^{2} \vec{x}_{i} / d t^{2}$, contact force $\vec{F}_{i}^{c}$, liquid bridge force $\vec{F}_{i}^{l}$, 164 gravitational force $m_{i} \vec{g}$, moment of inertia $I_{i}$, angular acceleration $d \vec{\omega}_{i} / d t$, angular velocity $\vec{\omega}_{i}$ and external moments resulting out of contact and liquid bridge forces $\vec{M}_{i}$. Explicit integration

investigated the mixing of wet particles in a bladed mixer application. Rotating drums with wet material were studied by Liu et al. [23] and Tsunazawa [22], who applied capillary bridge models and took viscosity effects into account. Further studies on this application were done by Washino et al. [24] who developed a new liquid dispersion model to take the partial wetting of particles into account. Heine et al. [59] investigated the droplet dynamics in the spray zone of a two-fluid nozzle and the single particle wetting with a coupled DEM-CFD approach. Furthermore, Lim et al. [60] studied mixtures under the influence of liquid in vibrated beds and He et al. [61] simulated wet cohesive particles in spout fluid bed applications. However, for screening such investigations are mostly lacking [13].

In addition to the liquid bridge models, an appropriate calibration of DEM parameters has to be performed to apply the DEM for complex processes like screening under the influence of moisture. A review for DEM parameters and contact models for granular material has been done by Horabik and Molenda [62] who highlighted the importance of material and interaction properties for obtaining reliable information out of DEM simulations. Several methods have been proposed to calibrate DEM parameters [63-66] and recently, a general straightforward procedure for spherical and non-spherical particles with a high degree of automation was proposed by Elskamp et al. [67].

\section{Numerical method}

In this section, the discrete element method and the applied force laws including the contact, the capillary and the viscous forces as well as the formation and rupture of liquid bridges are summarized.

\subsection{The discrete element method}

The DEM is capable of tracking the translational and rotational motion of particles in various systems $[15,68]$. For this purpose, the Newton's and Euler's equations are integrated schemes (comp. e.g. [69]) are used to solve both equations (eqs. $(1,2)$ ). 
A sketch of two colliding spheres of different sizes $i$ and $j$ is shown in Fig. 1. The contact forces consist of a normal component and the tangential forces

$$
\vec{F}_{i j}^{c}=\vec{F}_{i j}^{c n}+\vec{F}_{i j}^{c t}
$$

169 where the normal component is obtained from a linear spring damper model as

$$
\vec{F}_{i j}^{c n}=k^{n} \delta_{i j} \vec{n}_{i j}+\gamma^{n} \vec{v}_{i j}^{n}
$$

170

171

where $k^{n}$ is the spring stiffness, $\delta_{i j}$ the virtual overlap, $\vec{n}_{i j}$ a normal vector, $\gamma^{n}$ a damping coefficient and $\vec{v}_{i j}^{n}$ the normal velocity at the contact point [70].

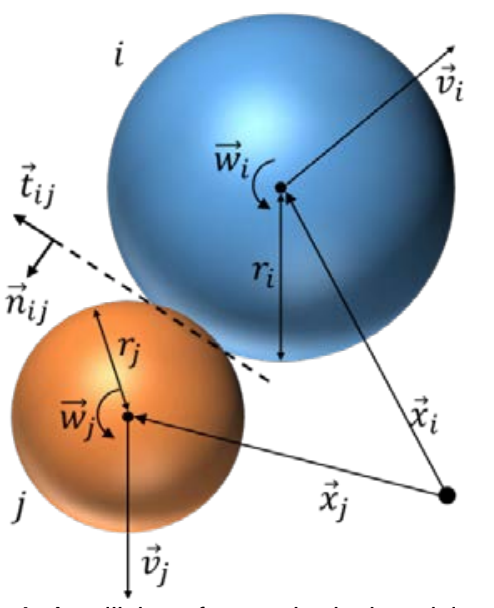

Fig. 1: A collision of two spherical particles

174 The coefficient of normal restitution between dry particles $e_{P P}^{n}$ as well as particles and walls $175 e_{P W}^{n}$ is determined by $k^{n}$ and $\gamma^{\mathrm{n}}$. For the tangential forces a linear spring limited by the

176 Coulomb condition is used, resulting in

$$
\vec{F}_{i j}^{c t}=-\min \left(k^{t}\left|\vec{\xi}_{i j}\right|, \mu_{C}\left|\vec{F}_{i j}^{n}\right|\right) \vec{t}_{i j}
$$

177 where $k^{t}$ is the tangential stiffness of a linear spring, $\mu_{C}$ is the friction coefficient, $\vec{\xi}_{i j}$ is the 178 relative tangential displacement and $\vec{t}_{i j}$ is the tangential unit vector [71].

\section{$179 \quad 2.2$ Liquid bridges in the discrete element method}

180 Liquid, which is added to dry material, can be in different states depending on the saturation 181 of the pores between the particles. If only a small amount of liquid is present, it will form 182 individual, pendular bridges between pairs of particles. In the funicular state more than two 183 particles can share one liquid bridge due to the filling of some of the pores between the 184 particles. In the capillary state all pores between the particles are filled with liquid [72]. In the 185 investigation here, only a small amount of liquid is added and uniformly distributed on the 186 particles to ensure the pendular state. 
The presence of liquid results in the formation of liquid bridges, which evokes several bonding

188 forces acting on the particles. In this investigation, only the capillary forces $\vec{F}_{i j}^{c a p}$ and the 189 viscous forces in normal $\vec{F}_{i j}^{n v i s}$ and tangential $\vec{F}_{i j}^{t v i s}$ direction are taken into account as

$$
\vec{F}_{i j}^{l}=\vec{F}_{i j}^{c a p}+\vec{F}_{i j}^{n v i s}+\vec{F}_{i j}^{t v i s}
$$

190

191

192

193

194

195

196

197

Note that the tangential capillary force vanishes due to the assumption of a symmetric structure of the liquid bridge. Moreover, the particle motion is not affected by the small mass of liquid in the liquid bridges (comp. [24]) and the gravitational force of the liquid is neglected, which is valid to assume for sufficiently small capillary bridges in the pendular state $[38,44]$. The external moment $\vec{M}_{i}$ (comp. eq. (2)) is extended and is now the sum of the moments due to a contact $\vec{M}_{C, i}$ and a liquid bridge $\vec{M}_{L, i}=\vec{r}_{i} \times \vec{F}_{i}^{t v i s}$.

\subsubsection{Liquid bridge formation and volume}

When two particles $i$ and $j$ or a particle and a wall get into contact under the influence of moisture, a liquid bridge forms out between the contact partners (comp. Fig. 2).

a

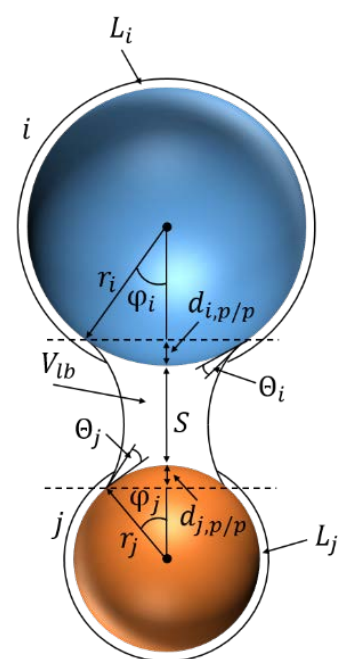

b

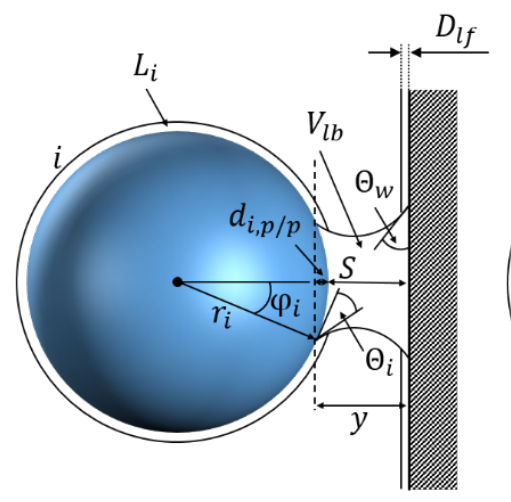

c

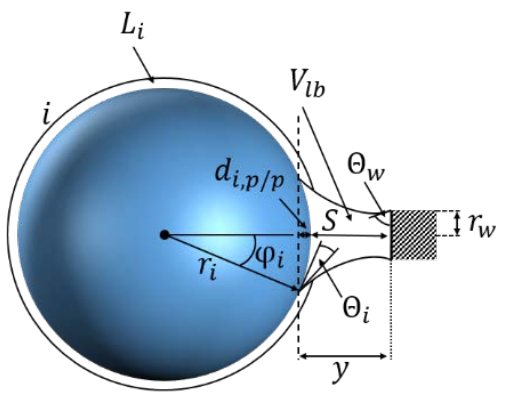

199

200

201

202

203

204

205

Fig. 2: Liquid bridges between (a) two different sized spherical particles, (b) a particle and a large wall (side wall, $r_{w}>r_{i}$ ) as well as (c) a particle and a small wall (screen wire, $r_{w}<r_{i}$ ).

In case of two particles, where a liquid with a low viscosity is equally distributed on their surfaces, the geometrical assumption is made, that the liquid on the spherical caps of the respective particles (dashed lines in Fig. 2) contributes in a liquid bridge (comp. [43]). The height of one of the spherical caps $d_{i, p / p}$ for particle $i$, also called immersion distance, is calculated as 


$$
d_{i, p / p}=r_{i}-\sqrt{r_{i}^{2}-\left(\frac{r_{i} r_{j}}{r_{i}+r_{j}}\right)^{2}}
$$

206

207

208

209

$$
V_{w}=W_{l f} \frac{\pi}{4} r_{i}^{2}
$$

For a wall, where one half side is shorter than the radius of the particle $\left(r_{w}<r_{i}\right.$, referred to as

with the radii $r_{i}$ and $r_{j}$ of the two particles $i$ and $j . d_{j, p / p}$ is calculated analogously. The total volume of the liquid bridge $V_{l b}$ is calculated for two spheres as proposed by Shi and McCarthy [43] as sum of the liquid volume contributed from each particle

$$
V_{l b}=V_{i}+V_{j}=\frac{L_{i}}{2}\left(1-\sqrt{1-\frac{r_{j}^{2}}{\left(r_{i}+r_{j}\right)^{2}}}\right)+\frac{L_{j}}{2}\left(1-\sqrt{1-\frac{r_{i}^{2}}{\left(r_{i}+r_{j}\right)^{2}}}\right)
$$

where $L_{i}$ and $L_{j}$ are the total liquid volumes present on particles $i$ and $j$. Note that the dimensionless liquid volume of the liquid bridge is $V_{l b}^{*}=V_{l b} / r_{e f f}^{3}$, where $r_{e f f}=2 r_{i} r_{j} /\left(r_{i}+r_{j}\right)$ is the effective radius. The assumption of an equally distributed liquid can be made for low liquid viscosities and hydrophilic surfaces [24] and has been proven as a suitable assumption in case that it is not desired to track the spatial distribution of the liquid on particles and walls [50]. Thereby, it should be noted, that it results in a slightly higher number of liquid bridges with respective lower volumes for materials with a low wettability compared to considering the spatial distribution of the liquid.

For a particle $i$ and a wall, the effective radius is $r_{e f f}=r_{i}$ and the volume of the liquid bridge is $V_{l b}=V_{i}+V_{w}$. In case of a wall, where the half length of the sides $\left(r_{w}\right)$ is larger than the radius $r_{i}$ of the sphere $\left(r_{w}>r_{i}\right.$, referred to as large wall) the liquid volume $V_{i}$ contributed from the particle is assumed to be like when a particle gets in contact with another particle of the same size, calculated as

$$
V_{i}=\frac{L_{i}}{2}(1-\sqrt{0.75})
$$

The liquid contributed from the wall is assumed as

where $W_{l f}$ is the liquid film thickness on the wall and $\frac{\pi}{4} r_{i}^{2}$ is the projection surface of the spherical cap of the particle on the wall (comp. Fig. 2). small wall), which is e.g. the case for screen wires, the liquid bridge volume is calculated differently. These wires are approximated by several triangular elements (comp. [11]), giving a nearly cylindrical shape. Two neighboring elements with the same normal vector form a flat surface. If a particle is close to a screen wire, a direct contact between the particle and one of 
these surfaces is assumed. The liquid contributed from the particle is based on the calculation of a spherical ring with the area $A_{s r}=2 \pi r_{i} 2 r_{w}$. The contact area of the sphere and a small wall is only the part $\frac{2 \varphi}{360}$ of this area, where $\varphi$ is the half filling angle, resulting in

$$
A_{s w}=2 \pi r_{i} 2 r_{w} \frac{2 \varphi}{360} .
$$

With proposed geometric considerations for equal sized spheres and a direct contact, it is $\varphi=$ $\cos ^{-1}\left(\frac{r_{i}-\mathrm{d}_{\mathrm{i}, \mathrm{p} / \mathrm{p}}}{r_{i}}\right)=30^{\circ}$. To obtain the liquid that contributes from the particle, the area $A_{s w}$ must be multiplied with the liquid film of the sphere which is $S_{l f}=\frac{L_{i}}{4 \pi r_{i}^{2}}$, giving

$$
V_{i}=\frac{L_{i}}{6 r_{i}} r_{w}
$$

Under the same geometric considerations, the liquid contributed from the small wall can be calculated as

$$
V_{w}=W_{l f} r_{i} 2 r_{w}
$$

where $r_{i} 2 r_{w}$ is the projection surface of the particle's spherical cap on the wall.

In this investigation, it is assumed that a liquid bridge between a particle and a wall is always located at the shortest distance of both contact partners. This means that the entire liquid bridge is moving with the particle and that it is not fixed at the first point of contact. Although a wall can be approximated by several triangular wall elements in the DEM, a particle is only able to have a liquid bridge contact with the closest element of this wall. If a particle is already in contact with another element of a wall, the existing contact information is transferred. Moreover, in this investigation the assumption is made, that the volume of the liquid bridge is constant from its formation until its rupture. The liquid volume from particles and walls contributing in liquid bridges is stored in temporary values until all liquid bridge formation processes of one time step are calculated. After that, the volume on the walls (proportional on each element of a wall) and on the particles is determined. This ensures that liquid bridge contacts of one contact partner with several other contact partners at the same time are all build up under the same conditions.

\subsubsection{Capillary liquid bridge force}

The capillary liquid bridge force can be calculated based on several different models. As described before, a classification can be made between the energetic method and a method based on the Young-Laplace equation. Additionally, the models can be subdivided into two groups the gorge (neck) and boundary (contact) methods. In the first group, the force is 
determined at the neck of the liquid bridge, whereas in the second group the force is calculated in the contact region of solid and liquid (comp. Fig. 2) [15]. A selection of models is briefly described in the following and later applied in DEM simulations.

Based on the models used by Rabinovich et al. [16] and Pitois et al. [44], which belong to the neck method, the capillary force between two particles $i$ and $j$ and between a particle $i$ and $a$ wall are calculated in this work as

$$
\begin{aligned}
& \vec{F}_{i j p p}^{c a p}=\left(-\frac{\pi \sigma r_{e f f}\left(\cos \theta_{i}+\cos \theta_{j}\right)}{1+1 /\left(\sqrt{1+\frac{2 V_{l i q}}{\left(\pi r_{e f f} S^{2}\right)}}-1\right)}-2 \pi \sigma r_{e f f} \sin \left(\theta_{i j}\right) \sin \left(\theta_{i j}+\varphi\right)\right) \vec{n}_{i j}, \\
& \vec{F}_{i p w}^{c a p}=\left(-\frac{2 \pi \sigma r_{i}\left(\cos \theta_{i}+\cos \theta_{w}\right)}{1+S \sqrt{\pi r_{i} / V_{l b}}}-2 \pi \sigma r_{i} \sin \left(\theta_{i w}\right) \sin \left(\theta_{i w}+\varphi\right)\right) \vec{n}_{i w},
\end{aligned}
$$

263 where $\sigma$ is the surface tension coefficient, $\theta_{i}, \theta_{j}$ and $\theta_{w}$ are the static contact angles of the 264 particles $i$ and $j$ and a wall, respectively. $\theta_{i j}=\left(\theta_{i}+\theta_{j}\right) / 2$ and $\theta_{i w}=\left(\theta_{i}+\theta_{w}\right) / 2$ are the mean contact angles between two particles as well as between a particle and a wall, respectively (comp. [73]). Note that the contact angle is the angle formed by a drop of liquid on the surface of a solid to the surface of this solid. The size of the contact angle between liquid and solid depends on the interaction between solid, liquid and vapor at the three phase contact points. The smaller this interaction is, the larger the contact angle becomes [74]. Therein, a differentiation is made between the dynamic contact angle, which occurs in case of wetting and dewetting of a solid as well as the static contact angle, where the surrounding does not

272 influence the contact area between liquid and solid during the measurement. Note that the 273 static contact angle is used for the applied models. $S$ is the separation distance between 274 particles or between particles and a wall. In the second part of both equations, the attraction 275 force due to the vertical component of the surface tension of the liquid bridge is taken into 276 account. Therein, the half filling or "embracing" angle $\varphi$ is calculated in case of two spheres as

$$
\varphi=\sqrt{S / r_{e f f}\left(-1+\sqrt{1+2 V_{l b} /\left(\pi r_{e f f} S^{2}\right)}\right)}
$$

277 The relation between the volume and a given half filling angle $\varphi$ is as follows

$$
V_{l b}=\pi \varphi^{2} r_{e f f}^{2} S+0.5 \pi \varphi^{4} r_{e f f}^{3}
$$

278 For a sphere and a plate with given $V_{l b}$, the relation is 


$$
\varphi=\sqrt{2 S / r_{i} \sqrt{1+V_{l b} /\left(\pi r_{i} S^{2}\right)}}
$$

279 In section 3, some other capillary bridge models, which are applicable for a liquid bridge 280 between two spheres are applied to validate them against experimental results and compare 281 them with the already introduced model by Rabinovich et al. [16]. Therefore, they are briefly 282 outlined here. In the capillary bridge model by Willett et al. [18] the force is calculated as

$$
\vec{F}_{i p p}^{c a p}=2 \pi r_{e f f} \sigma \exp \left(f_{1}-f_{2} \exp \left(f_{3} \ln S^{*}+f_{4} \ln ^{2} S^{*}\right)\right)
$$

283 where the scaled dimensionless half-separation distance is

$$
S^{*}=\frac{S}{2 \sqrt{V_{l b} / r_{e f f}}}
$$

284 and $f_{1}, f_{2}, f_{3}, f_{4}$ are coefficients derived by curve-fitting to a numerical solution. They are 285 functions of $\theta_{i j}$ and $V_{l b}^{*}$ which is explained in detail in the work by Willett et al. [18]. The latter 286 group of authors also proposed a simplified capillary bridge model where

$$
\vec{F}_{i p p}^{c a p}=\frac{2 \pi r_{e f f} \sigma\left(\cos \theta_{i}+\cos \theta_{w}\right)}{1+2.1 S^{*}+10 \cdot S^{* 2}}
$$

287 In the capillary bridge model by Weigert and Ripperger [19], which is an example for the 288 boundary method, the force is calculated as

$$
\vec{F}_{i p p}^{c a p}=\frac{\pi}{4}\left(2 r_{e f f}\right)^{2} p \sin ^{2} \varphi+2 \pi \sigma r_{e f f} \sin \left(\theta_{i j}\right) \sin \left(\theta_{i j}+\varphi\right)
$$

289 where the half filling angle can be obtained as

$$
\varphi=\sin ^{-1}\left(\frac{V_{l b}}{0.12\left(2 r_{e f f}\right)^{2}\left(1+6 S / 2 r_{e f f}\right)\left(1+1.1 \sin \left(\theta_{i j}\right)\right)}\right)^{0.25}
$$

290 and the capillary pressure is

$$
p=\sigma\left(\frac{\cos \left(\varphi+\theta_{i j}\right)}{r_{e f f}(1-\cos \varphi)+S}+\frac{1}{r_{e f f} \sin \varphi \frac{r_{e f f}(1-\cos \varphi)+S}{\cos \left(\varphi+\theta_{i j}\right)}\left(\sin \left(\theta_{i j}+\varphi\right)-1\right)}\right)
$$




\subsubsection{Viscous liquid bridge force}

292 The importance of the viscous force increases with a high liquid viscosity or larger interparticle 293 velocities [42]. Due to the high frequency motion of a screen apparatus, the latter is relevant 294 and therefore, this force is important in the following investigations. The normal viscous force 295 was derived by Adams and Perchard [42] and can be obtained by

$$
\vec{F}_{i j}^{n v i s}=-\frac{6 \pi \eta r_{r e f f}^{2} \vec{v}_{r}^{n}}{S}
$$

296 297 298 299 300

$$
\vec{F}_{i j}^{n v i s}=-\frac{6 \pi \eta r_{r e f f}^{2} \vec{v}_{r}^{n}}{S}\left(1-1 / \sqrt{\left(1+V_{l b} /\left(\pi r_{r e f f} S^{2}\right)\right)}\right)^{2} .
$$

301 The tangential viscous force is proportional to both the relative translational and rotational 302 velocities and is obtained by several authors $[24,36,43]$ as

$$
\vec{F}_{i j}^{t v i s}=-6 \pi \eta r_{r e f f}\left(\frac{8}{15} \ln \frac{r_{r e f f}}{S}+0.9588\right)\left(\vec{v}_{r}^{t}+\vec{\omega}_{r} \times \vec{n}_{i j}\right)
$$

303 with $\vec{v}_{r}^{t}=\vec{v}_{i}-\vec{v}_{j}-\vec{v}_{r}^{n}$ as the relative translational and $\vec{\omega}_{r}=r_{i} \vec{\omega}_{i}+r_{j} \vec{\omega}_{j}$ as rotational velocity of 304 305 the spheres. Based on the numerical solution of the stokes equation, Goldman et al. [45] proposed the following equation for the tangential viscous force

$$
\vec{F}_{i j}^{t v i s}=-6 \pi \eta r_{r e f f}\left(\frac{8}{15} \ln \frac{r_{r e f f}}{S}+0.9588\right) \vec{v}_{r}^{t}-6 \pi \eta r_{r e f f}\left(\frac{2}{15} \ln \frac{r_{r e f f}}{S}-0.2526\right) \vec{\omega}_{r} \times \vec{n}_{i j}
$$

306 which has a slight change in the part of the rotational velocity and is valid for smaller $S$ $307\left(S<0.1 r_{\text {reff }}\right)$. In case of large $S\left(S \geq 0.1 r_{\text {reff }}\right)$ the following equation is proposed by Goldmann et 308 al. [45]

$$
\begin{aligned}
& \vec{F}_{i j}^{t v i s}=-6 \pi \eta r_{\text {reff }}\left(\frac{8}{15} \ln \frac{r_{\text {reff }}}{S}+0.9588\right) \vec{v}_{r}^{t} \\
& -\frac{6 \pi \eta r_{r e f f}}{8}\left(\frac{r_{r e f f}}{S+r_{\text {reff }}}\right)^{4}\left(1-\frac{3 r_{r e f f}}{8\left(S+r_{r e f f}\right)}\right) \vec{\omega}_{r} \times \vec{n}_{i j} .
\end{aligned}
$$


When the separation distance $S$ approaches zero, the viscous forces tend to infinity. For this reason, a minimum separation distance $S_{\min }=0.001 r_{\text {reff }}$ is introduced and added to $S$ (comp. e.g. [47]).

\subsubsection{Liquid bridge rupture and redistribution}

At a respective distance between two particles or a particle and a wall, the liquid bridge

$$
S_{\text {rup }}=r_{e f f}\left(1+0.5 \theta_{i}\right) V_{l b}^{* 1 / 3}
$$

which is valid for equal contact angles. Willett et al. [18] extended this equation and calculated the rupture distance as

$$
S_{\text {rup }}=r_{e f f}\left(1+0.5 \theta_{i}\right)\left(V_{l b}^{* 1 / 3}+0.1 V_{l b}^{* 2 / 3}\right) \text {. }
$$

317 For different sized spheres and different contact angles, the rupture distance is dependent on 318 the contact angles and radii of the particles $r_{i}>r_{j}$ (comp. [18]) as

$$
S_{\text {rup }}=r_{e f f}\left(1+\left(0.125 \theta_{i}+0.125 \theta_{j}\right)\left(1+\frac{r_{j}}{r_{i}}\right)\right)\left(V_{l b}^{* 1 / 3}+\left(\frac{r_{j}}{2 r_{i}}-\frac{2}{5}\right) V_{l b}^{* 2 / 3}\right)
$$

319 In order to take into account the influence of the particle velocity on the rupture distance, Pitois 320 et al. [48] introduced the dynamic rupture distance as

$$
S_{\text {rup }, \text { dyn }}=S_{\text {rup }}\left(1+\sqrt{\left(\frac{\left(\vec{v}_{i}-\vec{v}_{j}\right) \eta}{\sigma}\right)}\right)
$$

321 When the bridge ruptures, it ruptures at its thinnest point and the liquid of the liquid bridge is redistributed on the contributing particles or the particle and the wall. Here, it is assumed, that the liquid is instantly added to the liquid amount of both contact partners without a local distribution. To ensure the same conditions for new liquid bridge contacts, the liquid amount of one time step is cumulated and added to the particle or wall at the end of the current time step (comp. section 2.2.1). The resulting liquid film thickness is calculated as

$$
P_{l f, i}=\sqrt[3]{\left(\frac{3 V_{l b}}{4 \pi}+r_{i}^{3}\right)}-r_{i}
$$

327 The liquid share, which is received by the particle or the wall, is dependent on the rupture 328 location. This location is dependent on the particle size, the contact angle and the volume of 
329 the liquid bridge. Note that due to neglecting the gravitational force for the liquid bridge, the 330 rupture location is not influenced by the vertical position of one particle to another one. If the 331 contributing particles are of the same size and have the same contact angles, the rupture 332 location is centered between them giving the same liquid amount for both contact partners. For 333 different sized particles with the same contact angles, which is the case in this study, the rupture location is closer to the small particle resulting in a larger amount of liquid assigned to the large one. To obtain this amount, the transfer ratio between the two contact partners is determined. Therefore, the shape of the liquid bridge before its rupture must be known, which can be assumed with the parabolic equation

$$
Y(x)=a x^{2}+b x+c
$$

338 The location of the thinnest point of this bridge is where the derivation $\mathrm{Y}^{\prime}(\mathrm{x})=0$ and is denoted 339 with the coordinates $\left(x_{\min }, y_{\min }\right)$. To obtain this point of the liquid bridge before its rupture, the 340 following six equations must be solved numerically. The three phase contact points are located 341 on the two spheres with the coordinates $(0, y(0))$ and $\left(d_{l b}, y\left(d_{l b}\right)\right)$ and can be obtained by

$$
\begin{aligned}
& y(0)=\sqrt{r_{i}^{2}-\left(r_{i}-d_{i, p / p}\right)^{2},} \\
& y\left(d_{l b}\right)=\sqrt{r_{j}^{2}-\left(r_{j}-d_{j, p / p}\right)^{2}}, \\
& d_{l b}=d_{i, p / p}+d_{j, p / p}+S,
\end{aligned}
$$

342 where $d_{l b}$ is the shortest length of the liquid bridge plus both cap heights. The solid liquid 343 contact angles are related to the previously described parameters as

$$
\begin{aligned}
& \theta_{i}=\frac{\pi}{2}+\tan ^{-1}\left(y^{\prime}(0)\right)-\sin ^{-1}\left(\frac{y(0)}{r_{i}}\right) \\
& \theta_{j}=\frac{\pi}{2}+\tan ^{-1}\left(y^{\prime}\left(d_{l b}\right)\right)-\sin ^{-1}\left(\frac{y\left(d_{l b}\right)}{r_{j}}\right) .
\end{aligned}
$$

344 The volume of the liquid bridge is

$$
V_{l b}=V_{l b, i}+V_{l b, j}
$$

345 where the volumes of the two parts of the liquid bridge, which are redistributed to each particle 346 after rupture, are given by 


$$
\begin{aligned}
& V_{l b, i}=\pi \int_{0}^{x_{\min }} y^{2}(x) d x-\frac{\pi}{6}\left(3 y^{2}(0) d_{i, p / p}+d_{i, p / p}^{3}\right), \\
& V_{l b, j}=\pi \int_{x_{\text {min }}}^{d_{l b}} y^{2}(x) d x-\frac{\pi b}{6}\left(3 y^{2}(0) d_{j, p / p}+d_{j, p / p}^{3}\right) .
\end{aligned}
$$

347 More details can be found in the works by Shi and McCarthy [43], Pepin et al. [49] and 348 Schmelzle and Nirschl [50].

349 In case of a particle and a large wall, similar equations have to be solved. It is assumed that 350 the shape of the bridge is the same as for two equal sized particles. Besides, the wall has no 351 spherical cap, so it is not subtracted from the liquid bridge volume, giving $V_{l b, w}=\pi \int_{x_{\min }}^{d_{l b}} y^{2}(x) d x$ (comp. eq. (43)). For a particle and a small wall, the shape is assumed to be like in case of two different sized particles where only the spherical cap of the particle is subtracted from the liquid bridge volume.

\section{3 "Intercell" liquid bridge particle contacts and parallelization in the DEM}

In addition to the implementation of the aforementioned models, it must be ensured, that the liquid bridge contacts are identified correctly in the applied DEM code. The following procedures are visualized in Fig. 3. In the DEM, it is essential to detect contacts between particles as well as between particles and walls fast and reliably. In order to avoid checking all possible contact partners of a domain for a contact, various detection methods based on Cartesian grids have been introduced, where based on binning of the particles only the possible contact partners in one cell are checked for a contact (comp. e.g. [75]). In a frequently used method, the grid is adjusted so that cells are larger than the largest applied particle diameter (comp. Fig. 3, the 8 large red, blue and white cells surrounded by red lines), and a particle is assigned to the cell where its center is located. In this way, possible contact partners are only in the same or surrounding cells. In case of a polydisperse system, many small particles can be in one cell, resulting in a longer time for the identification of contacts, giving a reason for a different grid based contact detection method. When relying on this approach throughout a DEM code, a small contact grid (comp. Fig. 3, the 16 small cells in each large cell) is applied to faster identify new and existing contacts between different sized particles (comp. [75]). A particle is assigned to each cell that is covered by a part of this particle.

372 Thereby, only cells with a particle assigned to it must be checked for a contact and the amount 373 of particles in one cell is comparatively small.

374 In case of a liquid bridge contact, the localization of a new bridge is similar but with the liquid 375 film added to the radius of the sphere as detection radius $r_{d}=P_{l f}+r_{i}$ (situation 1 in Fig. 3). 
Existing liquid bridges can become so large that they connect particles, which are not assigned to the same grid cell (situation 2 in Fig. 3). In order to detect these existing liquid bridges, a list containing the contact partners of each liquid bridge contact is created and checked each time step before the new liquid bridge contacts are identified. With the parameters $V_{l b}$ and $S_{\text {rup }}$ stored in a liquid bridge contact array, the liquid bridge forces, the rupture and the liquid redistribution can be calculated at each time step for each liquid bridge contact even for "intercell" liquid bridge particle contacts.

Process 1 Process 2

Domain cells 1 Boundary cells 1

Fig. 3: "Intercell" liquid bridge particle contacts and parallelization by domain decomposition in the DEM

384

385

386

387

388

389

390

391

392

393

394

395

396

397

398

399

400

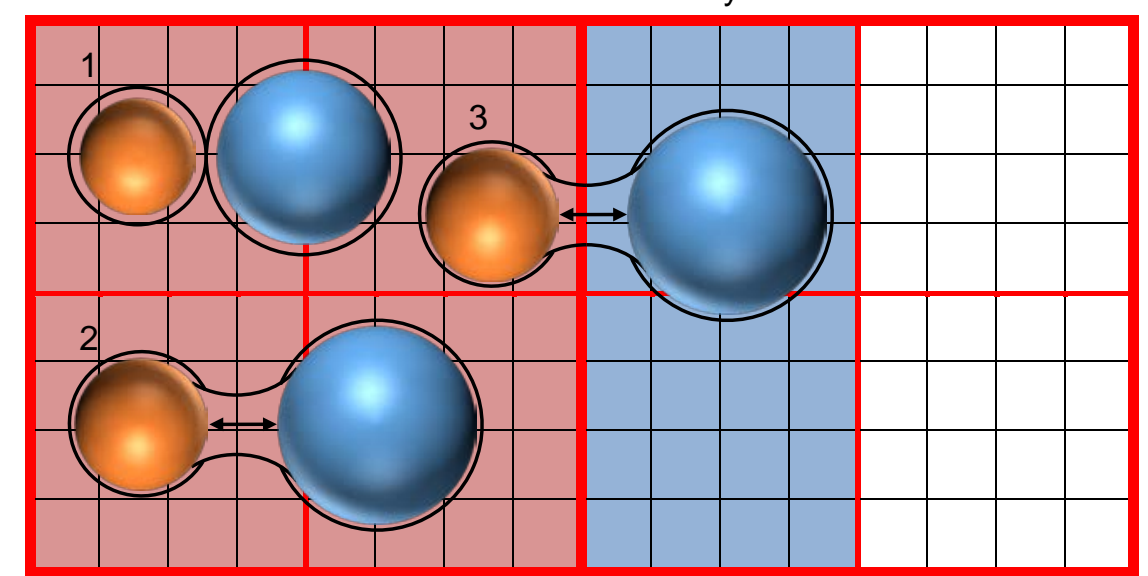

In order to require less computational time to perform DEM simulations of complex process steps involving large numbers of particles like screening multiple processors can be used. The applied DEM code is parallelized using domain decomposition, where the computational domain is divided spatially in several smaller domains (comp. Fig. 3, Process 1 (red) and Process 2 (blue, white)), each assigned to one processor (comp. e.g. [76]). By applying a uniform or possibly even load based division, the calculation time can theoretically decrease linearly with applied processors. An exchange of information between two neighboring domains is performed with the help of boundary cells of the large DEM grid described previously (comp. Fig. 3, large blue cells surrounded by red lines). The boundary cells are one layer of cells around the domain. Particles exist in their domain and as boundary particles in the neighboring domain, so that properties assigned to the particles and contacts between particles are known in both domains e.g. for the calculation of forces. To avoid duplications in the subsequent exchange between the domains and in the visualization, this information is saved after the calculation procedure of one time step and the boundary particles including its contacts are removed. In the next time step, the particles are again inserted in the boundary cells and the information is restored before the particles are repositioned by integrating the equations of motion. 
401 If parallel computing should be applied in the investigation here, the proper transfer of liquid 402 bridge contact information and its history over the process boundaries must be ensured 403 (situation 3 in Fig. 3). Therefore, the parts of the contact list containing liquid bridge contacts 404 between boundary particles and their contact partners in the domain, are transferred between 405 the processes. Additionally, the associated parts of the contact array with the stored liquid bridge contact data $\left(V_{l b}\right.$ and $S_{\text {rup }}$ ) and information about the boundary particles like the liquid volume on the particles $L_{i}$ are exchanged. The same procedure can be utilized when periodic boundaries are applied. Note that the cells of the DEM grid (large cells in Fig. 3) should be large enough to detect an existing liquid bridge contact for the largest possible liquid bridge in the respective simulation.

\section{Numerical validation}

In order to validate the implementation of the capillary liquid bridge forces in the DEM code, various simulations of two particles comprising of one single sphere in presence of a liquid bridge are conducted (comp. Fig. 5c). The simulation setup is similar to the one used in the work by Gladkyy and Schwarze [40] which corresponds to the experiments performed by Willet et al. [18] and Rabinovich et al. [16].

In the simulations, two spheres are placed in direct contact to each other $(S=0 \mathrm{~m})$, but without overlapping to prevent contact forces. This results in the formation of a liquid bridge. Gravitational forces are not taken into account. One particle is pulled away slowly to avoid viscous effects while the other one is fixed. The capillary liquid bridge force $F_{\text {cap }}$ is tracked until the bridge brakes due to reaching the rupture distance $S_{\text {rup }}$. The implementation for a particle and a wall is validated similarly. Therefore, a particle is placed in direct contact to a wall to form out a liquid bridge before it is slowly pulled away from the wall.

In the work by Willet et al. [18] precision synthetic sapphire spheres and dimethylsiloxane as fluid with a surface tension of $\sigma=20.6 \mathrm{mN} / \mathrm{m}$ and a contact angle of $\theta_{i}=\theta_{j}=0^{\circ}$ are used. Rabinovich et al. [16] used smaller silica particles, different oils with surface tensions of $\sigma=24-28 \mathrm{mN} / \mathrm{m}$ and a contact angle between particles of $\theta_{i}=\theta_{j}=10^{\circ}$ and between a particle and a wall of $\theta_{i}=\theta_{w}=0-10^{\circ}$.

In Fig. 4 exemplary results for the calculation of the capillary liquid bridge forces in the simulations for four different models $[16,18,19]$ are compared to the corresponding experimental data by Willet et al. [18] (Fig. 4a) and Rabinovich et al. [16] (Fig. 4b). In Fig. 4a, the results for a liquid bridge of $V_{l b}=13.6^{\star} 10^{-12} \mathrm{~m}^{3}$ between particles of $r_{1}=r_{2}=2.381 \mathrm{~mm}$ are presented. The models by Willet et al. [18] and Rabinovich et al. [16] fit the experimental results well over the whole distance and reveal only deviations for very small distances. In contrast, 
the model by Weigert and Ripperger [19] provides the best result for a very small distance but has large discrepancies for larger distances. Fig. 4b shows the results for a liquid bridge of $V_{l b}=2 * 10^{-19} \mathrm{~m}^{3}$ between smaller particles of $r_{1}=19 \mu \mathrm{m}$ and $r_{2}=35 \mu \mathrm{m}$. The results are similar to those obtained for larger particles and volumes.

a

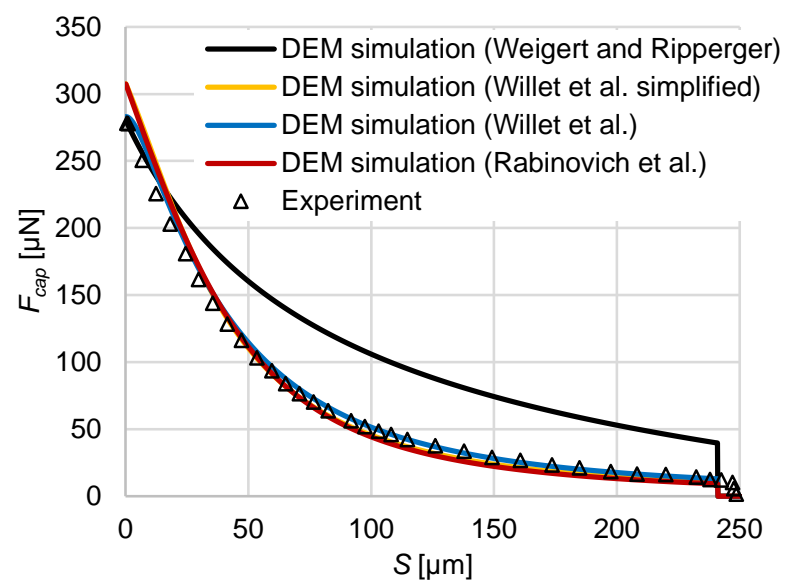

b

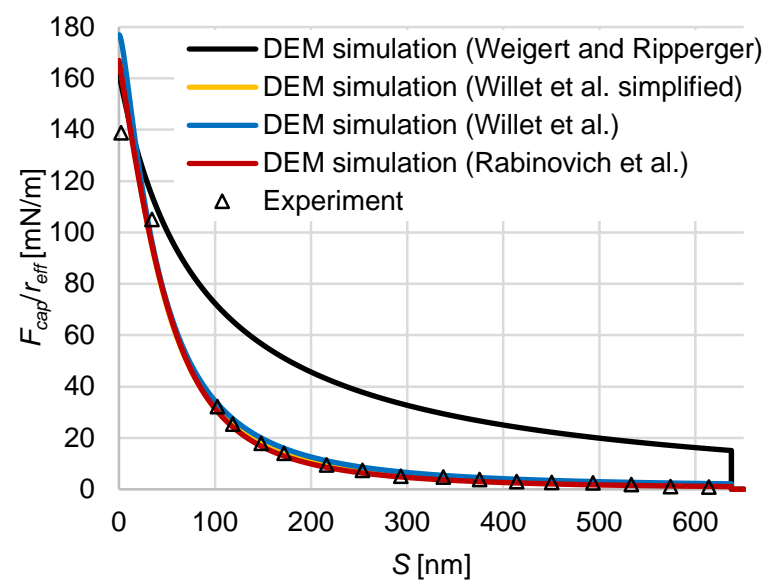

439

440

441

442

443

444

445

446

447

448

449

450

451

452

453

454

455

456

457

458

459

460

461

Fig. 4: Comparison of capillary liquid bridge forces of various models in DEM simulations to corresponding experimental data by (a) Willet et al. [18] for a liquid bridge of $V_{l b}=13.6^{*} 10^{-12} \mathrm{~m}^{3}$ between particles with $r_{1}=r_{2}=2.381 \mathrm{~mm}$ and $\theta_{i}=\theta_{j}=0^{\circ}$ as well as by (b) Rabinovich et al. [16] for a liquid bridge of $V_{l b}=2^{*} 10^{-19} \mathrm{~m}^{3}$ between particles with $r_{1}=19 \mu \mathrm{m}, r_{2}=35 \mu \mathrm{m}$, and $\theta_{i}=\theta_{j}=0^{\circ}$.

The results for a liquid bridge contact between a particle and a wall are presented in Fig. $5 \mathrm{a}$ only for the model by Rabinovich et al. [16] because the other models are not directly applicable for particle wall contacts. The applied parameters are $r_{1}=12 \mu \mathrm{m}, V_{l b}=7^{\star} 10^{-19} \mathrm{~m}^{3}, \theta_{i}=\theta_{w}=0^{\circ}$ for the first configuration and $r_{1}=25 \mu \mathrm{m}, V_{l b}=170^{\star} 10^{-19} \mathrm{~m}^{3}, \theta_{i}=\theta_{w}=10^{\circ}$ for the second configuration. The surface tension is $\sigma=26 \mathrm{mN} / \mathrm{m}$ in both cases. Besides minor deviations at very small distances, the model by Rabinovich et al. [16] fits the experimental results very well in both cases. Therefore, this model is applied in the DEM simulations for the capillary forces. For the validation of the implementation of the viscous liquid bridge forces in the DEM code a similar setup as for the capillary forces is used, which corresponds to the experiments performed by Pitois et al. [48] (comp. Fig. 5c). In these experiments polished ruby spheres of $r_{1}=r_{2}=4 \mathrm{~mm}$ with a contact angle of $\theta_{i}=\theta_{j}=10^{\circ}$ are applied and one particle is pulled away with a constant velocity which is changed for the different experiments (here $v=0.1-10 \mathrm{~mm} / \mathrm{s}$ ). In the investigation here, a configuration with liquid properties of $V_{l b}=5^{\star} 10^{-10} \mathrm{~m}^{3}, \sigma=21 \mathrm{mN} / \mathrm{m}$ and $\eta=0.1 \mathrm{~kg} / \mathrm{ms}^{2}$ is simulated and compared to the experiments. In the simulations, the capillary and viscous liquid bridge forces are recorded as total liquid bridge force $F_{\text {tot }}=F_{\text {cap }}+$ $F_{\text {vis }}$ until the bridge brakes due to reaching the rupture distance $S_{\text {rup. }}$

In Fig. $5 b$, the dimensionless force $F_{\text {tot }} / \sigma r_{\text {eff }}$ is plotted over the dimensionless distance $S / r_{\text {eff. }}$. Note that the capillary model by Rabinovich et al. [16] is applied for calculating the capillary forces and the viscous models by Pitois et al. [44] and Goldmann et al. [45] are used for the viscous forces. In case of a low velocity of $v=0.1 \mathrm{~mm} / \mathrm{s}, F_{\text {tot }}$ is nearly completely determined 

distances between the particles. The simulation results fit the experimental ones very well for all applied velocities.

a

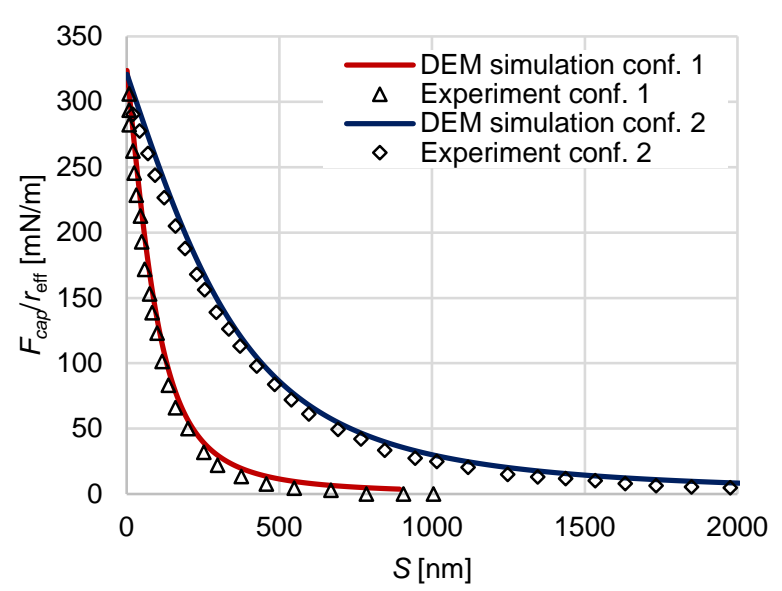

b

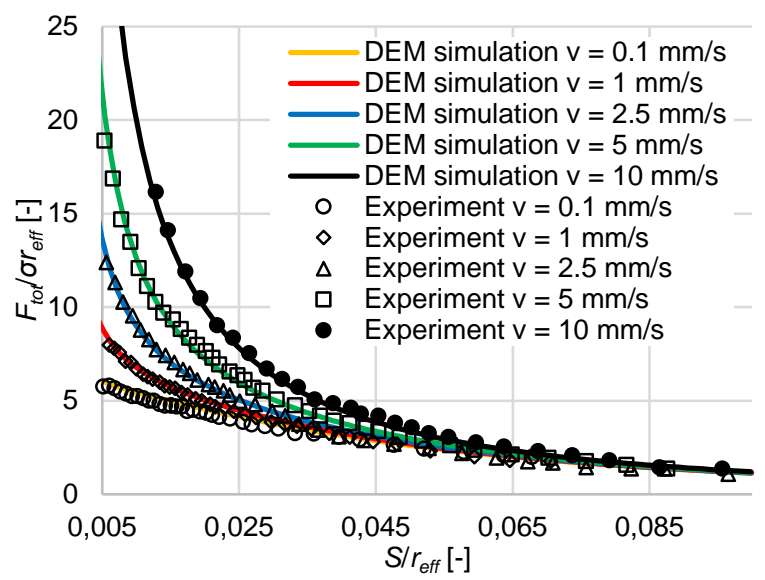

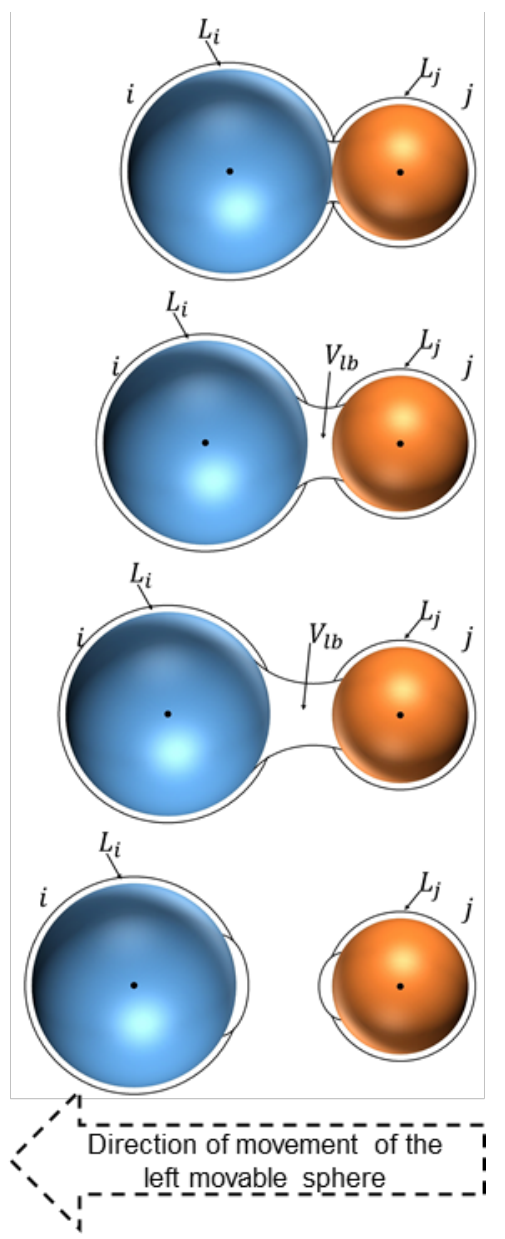

Fig. 5: (a) Comparison of capillary liquid bridge forces in DEM simulations to corresponding experimental data by Rabinovich et al. [16] for a liquid bridge between a wall and a particle with conf. 1: $r_{i}=12 \mu \mathrm{m}, V_{l b}=7^{\star} 10^{-19} \mathrm{~m}^{3}$ and $\theta_{i}=\theta_{w}=0^{\circ}$ as well as conf. 2 : $r_{i}=25 \mu \mathrm{m}, V_{l b}=170^{\star} 10^{-19} \mathrm{~m}^{3}$ and $\theta_{i}=\theta_{w}=10^{\circ}$. (b) Comparison of liquid bridge forces (capillary and viscous) in DEM simulations to corresponding experimental data by Pitois et al. [48] for a liquid bridge of $V_{l b}=5^{*} 10^{-10} \mathrm{~m}^{3}$ between particles with $r_{1}=r_{2}=4 \mathrm{~mm}$ and $\theta_{i}=\theta_{j}=10^{\circ}$ as well as various constant particle velocities. (c) Schematic representation of the procedure until the bridge ruptures.

\section{Determination of the contact angle and the transfer ratio}

As previously described, larger contact angles reduce the capillary liquid bridge force and extend the rupture distance. In addition, a lower contact angle of one contact partner results in more liquid redistributed on this contact partner after the liquid bridge ruptures. Therefore, it is important to obtain the contact angle for the applied particles and walls with the respective liquid. In this work, polyoxymethylene (POM) and glass spheres are used for the particles as well as stainless steel and treated PVC (polyvinylchlorid) for the walls and water with a surface tension of $\sigma=72.75 \mathrm{mN} / \mathrm{m}$ as liquid. 


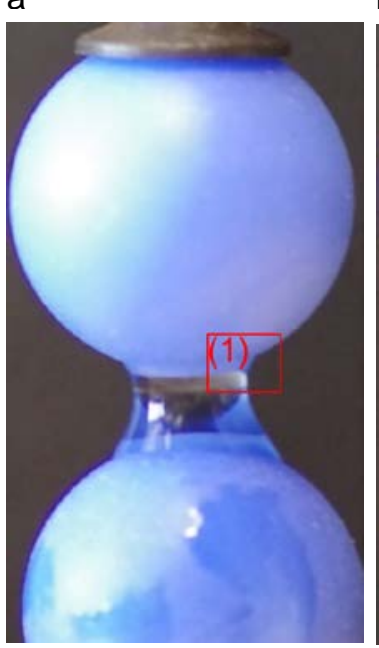

b

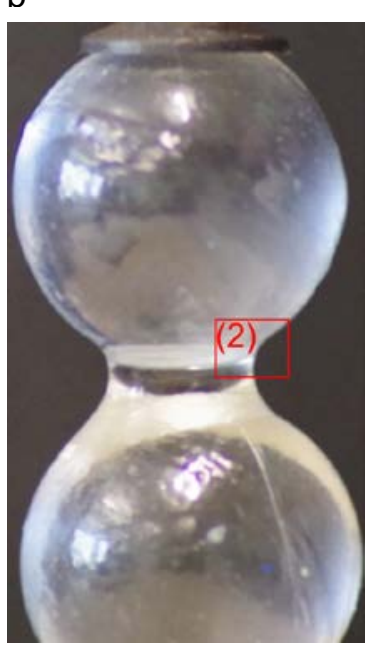

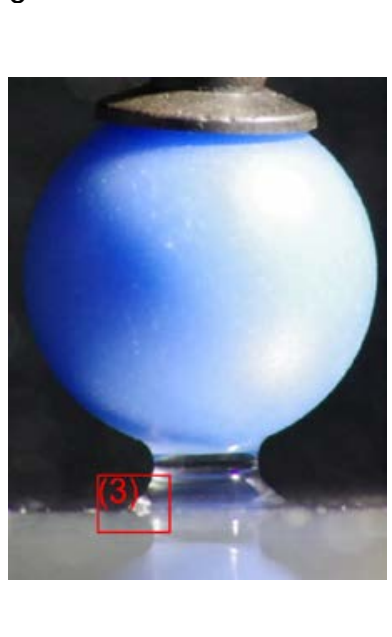

d

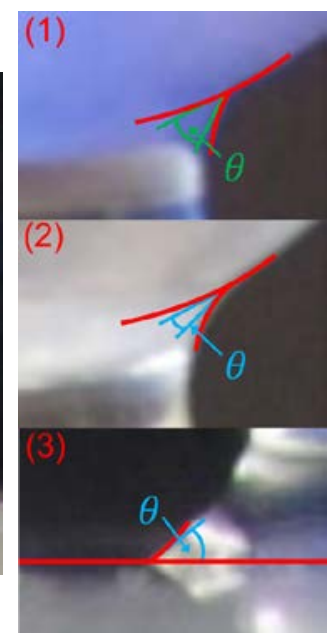

483

484

485

486

487

488

489

490

491

492

493

494

495

496

497

498

499

500

501

502

503

Fig. 6: Liquid bridges between (a) POM spheres, (b) glass spheres and (c) a sphere and a steel plate. (d) Close-up of the contact angles

In the investigation here, an optical measurement tool is utilized to measure the angle between the slope of the liquid bridge and the tangent to the sphere at the three phase contact points (comp. Fig. 6). The average value of 10 experiments is determined. It can be seen in Fig. $6 \mathrm{~d}$ that the contact angle between the POM spheres and water (1) is much larger than between the glass spheres and water (2). Furthermore, the bridge between the glass spheres is wider, giving a smooth transition between sphere and liquid. Note that in this investigation the static contact angle is measured and applied in the DEM. In case of two spheres, the contact angle between each single spheres and the water was measured and the average value was taken to account for gravitational effects. This is due to reducing the complexity for processes with many particle and wall elements. In addition, the applied capillary force, rupture and redistribution models are based on the static contact angle (comp. e.g. [51]).

With the obtained contact angles (comp. Table 1), it is now possible to determine the liquid volumes $V_{l b, i}$ and $V_{l b, j}$ assigned to the particles $i$ and $j$ after the rupture of a liquid bridge by numerically solving the equations given in section 2.2.4. With these liquid volumes, the transfer ratio $T_{r, i}=V_{l b, i} /\left(V_{l b, i}+V_{l b, j}\right)$ between contact partners can be obtained. Note that the transfer ratio is related to particle $i$, whereas the ratio for particle $j$ is $T_{r, j}=1-T_{r, i}$. In Figs. $7 a, b$ the transfer ratios for several particle sizes and two contact angle combinations are shown for dimensionless liquid volumes of $V_{l b}^{*} \leq 0.5$. The transfer ratio increases for a larger particle size difference $r_{i} / r_{j}$ and larger $V_{l b}^{*}$. For a low contact angle (comp. Fig. 7a), the transfer ratio inclines 
nearly linearly and slowly with $V_{l b}^{*}$, whereas a larger contact angle (comp. Fig. $7 \mathrm{~b}$ ) results in a steeper increase. Nevertheless, the transfer ratio for contact angles of $\theta_{i}=\theta_{i}=10^{\circ}$ is lower than for $\theta_{i}=\theta_{i}=40^{\circ}$ for $V_{l b}^{*} \leq 0.4$.

507 The transfer ratio between a particle and a wall is presented in Figs. 7c,d for several contact angle combinations. Here, the contact angle between a sphere and the water is chosen as before $\left(\theta_{i}=10^{\circ}\right.$ in Fig. $7 \mathrm{c}$ and $\theta_{i}=40^{\circ}$ in Fig. $\left.7 \mathrm{~d}\right)$. As expected, a larger $\theta_{w}$ results in a higher transfer ratio $T_{r, i}$ and a larger $\theta_{i}$ causes a lower $T_{r, i}$. Additionally, if $\theta_{i}<\theta_{w}$ the transfer ratio 511 increases with $V_{l b}^{*}$ and if $\theta_{i}>\theta_{w}$ it declines.

a

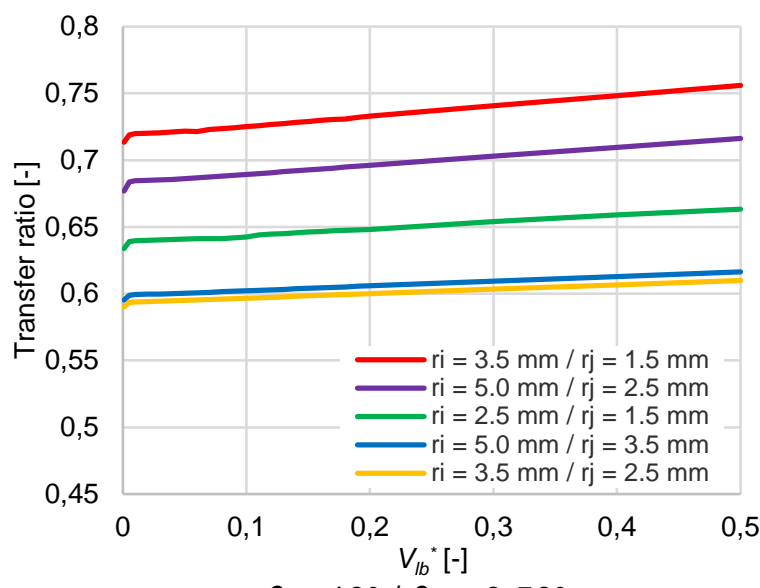

C

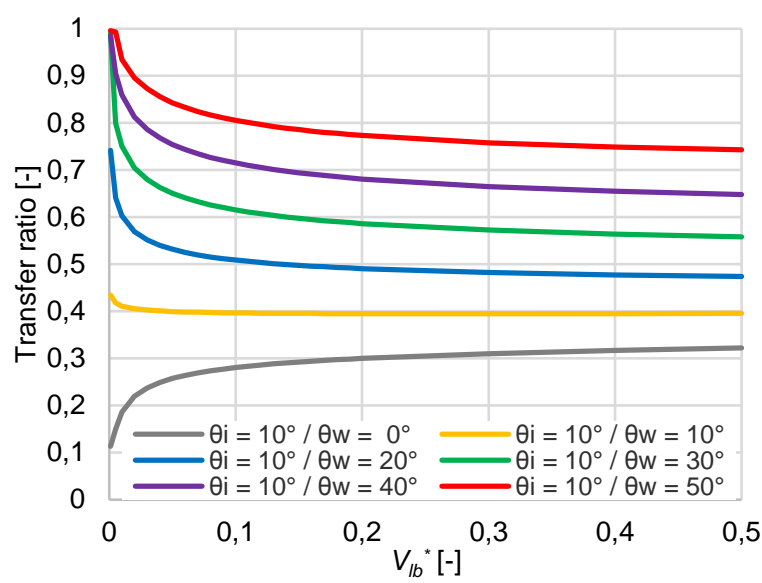

b $\quad \theta_{i}=40^{\circ} / \theta_{j}=40^{\circ}$

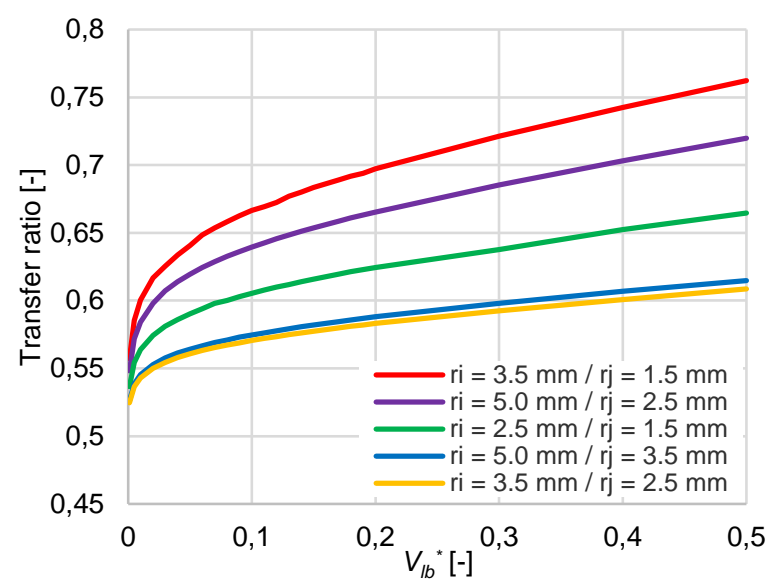

$\theta_{i}=40^{\circ} / \theta_{w}=0-50^{\circ}$

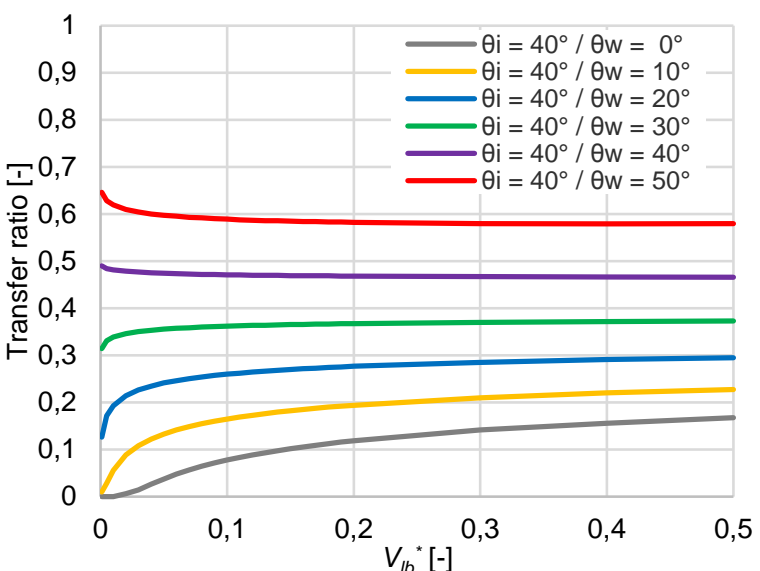

Fig. 7: Transfer ratio between spheres of different sizes and a contact angle of (a) $\theta_{i}=\theta_{j}=10^{\circ}$ and (b) $\theta_{i}=\theta_{j}=40^{\circ}$ as well as spheres of $r_{i}=1.5 \mathrm{~mm}$ with a contact angle of (c) $\theta_{i}=10^{\circ}$ and (d) $\theta_{i}=40^{\circ}$ and different walls with contact angles of $\theta_{w}=0-50^{\circ}$.

514 An equation for the transfer ratio, which depends on the particle size and the liquid bridge

515 volume, cannot be easily obtained to be applied in DEM simulations. Hence, for the DEM code,

516 a look-up table is generated to include this data with minimal more computational effort. This 517 look-up table is created in Matlab ${ }^{\circ}$ for various possible contact partners before it is used in 518 DEM simulations. Just one time in the initialization process of a DEM simulation this look-up 519 table is read and only the required data (transfer ratios for all applied contact angle and contact partner combinations for liquid volumes of $\left.V_{l b}^{*} \leq 0.5\right)$ is written in a transfer ratio array. If 
521 necessary, non-existing values are obtained by linearly interpolating between available data.

522 When a bridge ruptures during the DEM simulation, the transfer ratio for the respective contact

523 angle and contact partner combination as well as the current liquid bridge volume (interpolation

524 required) is obtained with the data stored in this transfer ratio array.

\section{5. Experimental and numerical batch screening}

526 A validation of the used DEM code and an in depth investigation of the related subprocesses 527 during screening and their linkage to liquid bridge formation, stressing and rupture as well as 528 liquid redistribution is performed by a comparison of results obtained from batch screening 529 experiments and simulations under the influence of various liquid amounts.

\section{$530 \quad 5.1$ Experimental and numerical setup}

531 The mechanical and physical particle and wall properties are presented in Table 1. For the 532 experiments in this study, a batch screening apparatus, which can be applied for dry and wet 533 screening (comp. Fig. 8) is used. The screen apparatus is a modified "Haver and Boecker EML 534 digital plus" batch screen tower with a circular screen surface, additionally equipped with a 535 feed bin on top of it to ensure that the particles in experiment and simulation reach the screen 536 surface at the same time and that the screen excitation is already in a continuous motion. 537 Additionally, an outlet is added below the screen to measure the particle passage through the 538 apertures when they reach the collecting bin on a balance. Various screens with different 539 aperture sizes can be staked over the outlet of the screen apparatus. In the investigation here, 540 one screen surface is applied in each case with the aperture sizes in Table 2 adjusted to the 541 particle sizes in Table 1.

542 Table 1: Mechanical and physical particle and wall properties.

\begin{tabular}{c|c|c|cc}
\hline & \multicolumn{2}{|c|}{ Particle } & Wall \\
\hline Mechanical particle property & POM & Glass & Steel & PVC \\
\hline Diameter $d[\mathrm{~mm}]$ & $3 / 5 / 7 / 10 \pm 0.1$ & $3 / 5 / 7 / 10 \pm 0.1$ & - \\
Mass $m[\mathrm{~g}]$ & $0.0192 / 0.0935 / 0.2459 /$ & $0.0353 / 0.1636 / 0.4490 /$ & - \\
& $0.7210 \pm 0.02$ & $1.3090 \pm 0.02$ & \\
Density $\rho\left[\mathrm{kg} / \mathrm{m}^{3}\right]$ & $1.3570 \mathrm{E}+03 / 1.3580 \mathrm{E}+03 /$ & $2.5240 \mathrm{E}+03 / 2.5351 \mathrm{E}+03 /$ & & \\
& $1.3356 \mathrm{E}+03 / 1.3425 \mathrm{E}+03$ & $2.5373 \mathrm{E}+03 / 2.5300 \mathrm{E}+03$ & $7.85 \mathrm{E}+03$ & $1.30 \mathrm{E}+03$ \\
Young's modulus $E\left[\mathrm{~N} / \mathrm{m}^{2}\right]$ & \pm 1.50 & \pm 1.50 & $2.08 \mathrm{E}+11$ & $2.20 \mathrm{E}+09$ \\
Poisson's ratio $v[-]$ & $2.84 \mathrm{E}+09$ & $5.00 \mathrm{E}+10$ & 0.4 \\
Stiffness $k^{n}{ }_{P P} / k^{n}{ }_{P W}[\mathrm{~N} / \mathrm{m}]$ & 0.35 & 0.2 & 0.30 & - \\
Contact angle $\theta\left[{ }^{\circ}\right]$ & $1.00 \mathrm{E}+05$ & $1.00 \mathrm{E}+05$ & - & 50 \\
\hline
\end{tabular}




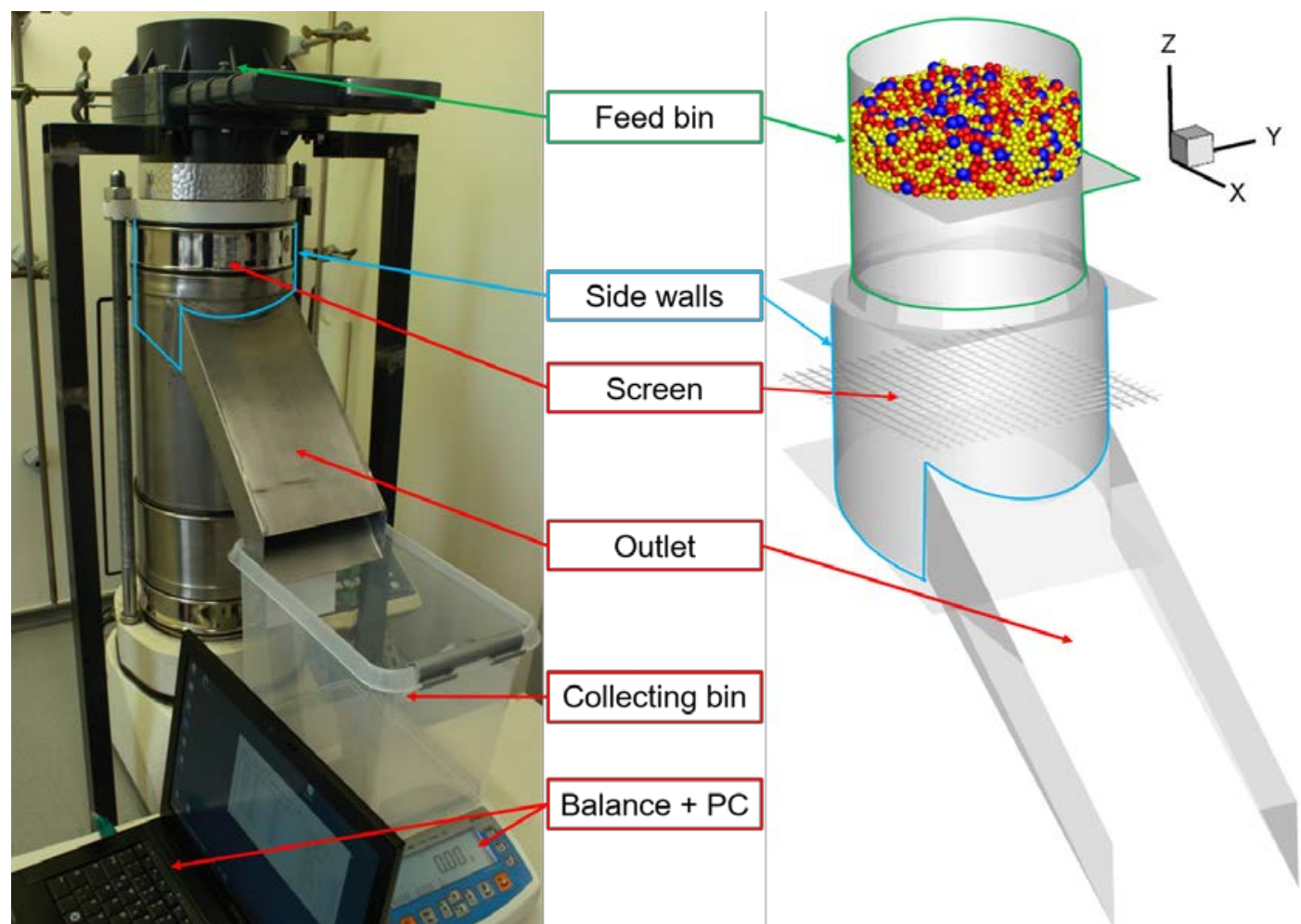

544

545

546

547

548

549

550

551

552

553

554

555

556

557

558

559

560

561

562

563

564

Fig. 8: (a) Experimental setup and (b) corresponding approximation in the DEM simulations of the batch screening apparatus. In a first step, the weighted particles are filled well mixed with the respective already attached amount of water in the feed bin on a flat surface. After the screen reaches a steady motion, the surface in the feed bin is pulled out and all particles drop as bulk material on the screen surface. Some smaller particles directly pass through the apertures while others have to stratify through the gaps between larger ones until getting the possibility to pass through the apertures. After that, the particles drop on an inclined impact plate from where they get to the outlet and through it on a balance, which continuously weights the incoming material. In this way, the remaining mass over time can be compared between experiment and simulation for various configurations.

In this investigation, POM and glass spheres are applied in three different equally distributed discrete size classes. They are assumed to be ideal spheres of $d_{1}=5 \mathrm{~mm}, d_{2}=7 \mathrm{~mm}$, $d_{3}=10 \mathrm{~mm}$ in the first configuration and $d_{1}=3 \mathrm{~mm}, d_{2}=5 \mathrm{~mm}$ and $d_{3}=7 \mathrm{~mm}$ in the second configuration. In all configurations, the particles and the aperture size are in the same relationship, $d_{1}<d_{2}<a<d_{3}$. In the following the particle classes are called small $\left(d_{1}\right)$, near mesh $\left(d_{2}\right)$, which has the additional relationship $0.8 \mathrm{a}<d_{2}<a$, and large $\left(d_{3}\right)$. POM spheres are filled into the feed bin with a mass of $m_{p}=3 m_{p i}=3 \times 250 \mathrm{~g}=750 \mathrm{~g}$. The amount of glass spheres is chosen to be volume equivalent with the POM spheres giving a mass of $m_{p} \approx 1410 \mathrm{~g}$ (comp. Table 2).

The experimental properties for the batch screening experiments can be found in Table 2. For both materials, three different liquid amounts in the range of $0 \% \leq M \leq 10 \%$ are applied. In 
565 the case of glass spheres, the percentage amount is lower in order to maintain a pendular 566 regime. The applied liquid is distilled water. At the beginning of each simulation, the same 567 liquid film thickness $P_{l f}$ is assigned to each particle (comp. [22]). The walls are dry $\left(V^{*}=0\right)$.

568 The screen profile is woven with cylindrical wires of $w=2 \mathrm{~mm}$ and $w=1.6 \mathrm{~mm}$ giving aperture 569 sizes of $a=8 \mathrm{~mm}$ and $a=5.6 \mathrm{~mm}$, respectively. Note that the wires in the DEM simulation are 570 approximated as horizontal bars (not woven) with a cylindrical profile, which has proven to be 571 a valid simplification (comp. [11]).

572 Table 2: Experimental properties for batch screening experiments.

\begin{tabular}{c|cc}
\hline Properties & POM & Glass \\
\hline Particle mass $[\mathrm{kg}]$ & 0.75 & $\sim 1.41$ \\
Liquid amount [\%] & $0 / 5 / 10$ & $0 / 2.5 / 5$ \\
Surface tension $[\mathrm{N} / \mathrm{m}]$ & \multicolumn{2}{|c}{0.07275} \\
Aperture size $[\mathrm{mm}]$ & $8.00 \pm 0.02 / 5.60 \pm 0.01$ \\
Wire diameter [mm] & $2.00 \pm 0.01 / 1.60 \pm 0.01$ \\
Scree wire profile [-] & Cylindrical (woven) \\
Set amplitude $[\mathrm{mm}]$ & \multicolumn{2}{|c}{1 / 0.8 } \\
Frequency [Hz] & $\sim 50.6$ \\
Stroke behavior & Elliptical, mainly vertical (comp. Fig. 9) \\
\hline
\end{tabular}

573

574 In Fig. 9, the screen motion in 3D obtained by measurements of an accelerometer ("Sequoia 575 FastTracer PA") fixed under the screen is presented. The amplitude is set to $A=1 \mathrm{~mm}$ and to $576 A=0.8 \mathrm{~mm}$, respectively, resulting in a frequency of approximately $f=50.6 \mathrm{~Hz}$. The motion is 577 elliptical but mainly in vertical direction (z-direction of the screen of about $0.9 \mathrm{~mm}$ and $0.72 \mathrm{~mm}$ 578 for the different configurations, respectively) while the motion in $x$ - and $y$ - directions is low with 579 maximum amplitudes of $A<0.1 \mathrm{~mm}$. In the following, the set amplitude is used to differentiate 580 the cases.

a

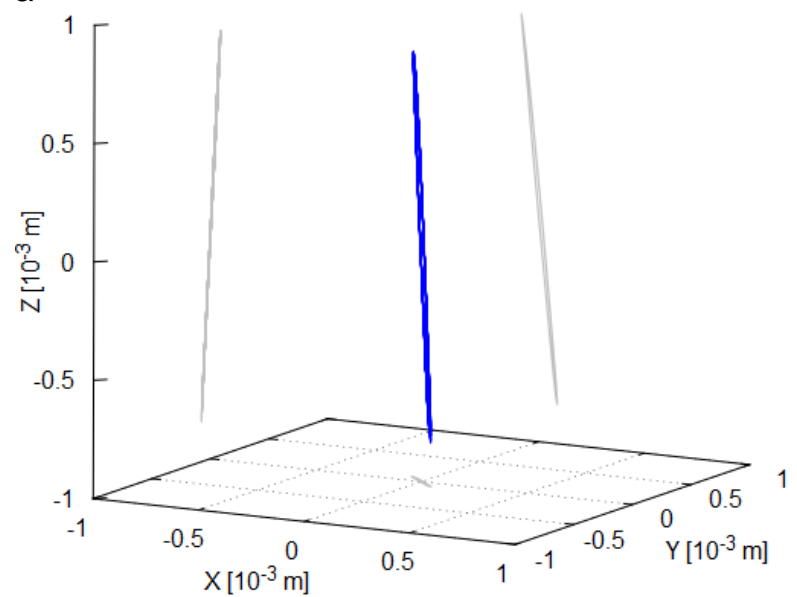

b

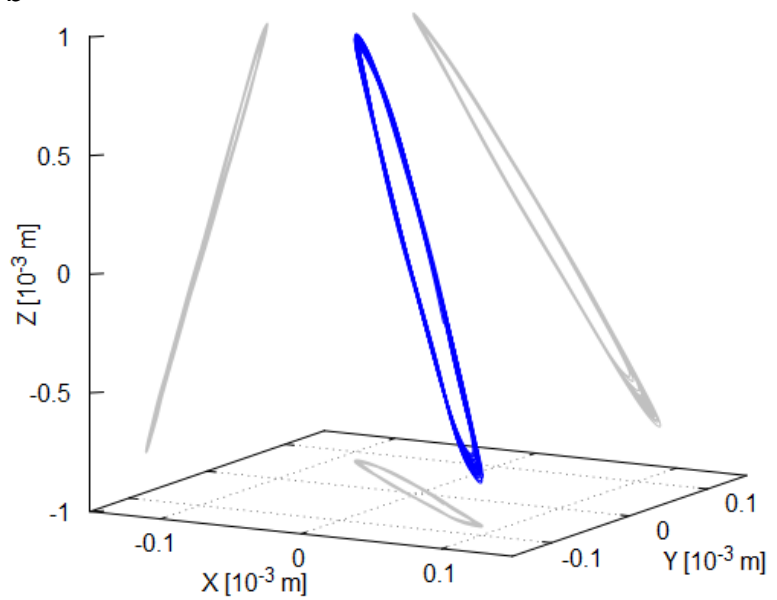


583 In Table 3 the DEM parameters coulomb friction $\mu_{c}$, rolling friction $\mu_{\text {roll }}$ and the coefficient of 584 restitution $e^{n}$ for POM and glass spheres with their respective contact partners are listed.

585 Details according the determination can be found in a previous work by the authors [67]. Note 586 that the coefficient of restitution for dry particles was obtained and applied in the simulations 587 due to considering the adhering effects by implementing the liquid bridge models.

588 Table 3: DEM parameters for POM and glass spheres and various contact partners

\begin{tabular}{c|c|ccc}
\hline Contact partner 1 & Contact partner 2 & $\mu_{c}[-]$ & $\mu_{\text {roll }}[\mathrm{m}]$ & $e_{d r y}^{n}[-]$ \\
\hline POM sphere & $\begin{array}{c}\text { Steel (side walls, screen wires, bottom, } \\
\text { outlet walls) }\end{array}$ & 0.3484 & $5.97 \mathrm{E}-05$ & 0.8473 \\
POM sphere & POM sphere & 0.3725 & $4.63 \mathrm{E}-05$ & 0.8038 \\
Glass sphere & $\begin{array}{c}\text { Steel (side walls, screen wires, bottom, } \\
\text { outlet walls) } \\
\text { Glass sphere }\end{array}$ & 0.2866 & $1.09 \mathrm{E}-04$ & 0.4351 \\
\hline
\end{tabular}

\subsection{Fraction retained in experiments and DEM simulations}

591 In the following, a comparison of the fraction retained between experiments and DEM 592 simulations is carried out. An overview of all performed experiments and DEM simulations can 593 be found in Table 4.

594 Table 4: Overview of performed simulations and experiments (averaged over 15 experiments)

\begin{tabular}{cccccc}
\hline $\begin{array}{c}\text { Simulation } \\
\text { No. }\end{array}$ & Material & $\begin{array}{c}\text { Particle size } \\
d_{1 / 2 / 3}[\mathrm{~mm}]\end{array}$ & $\begin{array}{c}\text { Aperture size } \\
{[\mathrm{mm}]}\end{array}$ & $\begin{array}{c}\text { Amplitude } \\
A[\mathrm{~mm}]\end{array}$ & $\begin{array}{c}\text { Liquid amount } \\
M[\%]\end{array}$ \\
\hline 1 & POM & $5 / 7 / 10$ & 8 & 1 & 0 \\
2 & POM & $5 / 7 / 10$ & 8 & 1 & 5 \\
3 & POM & $5 / 7 / 10$ & 8 & 1 & 10 \\
4 & POM & $5 / 7 / 10$ & 8 & 0.8 & 0 \\
5 & POM & $5 / 7 / 10$ & 8 & 0.8 & 5 \\
6 & POM & $5 / 7 / 10$ & 8 & 0.8 & 10 \\
7 & POM & $3 / 5 / 7$ & 5.6 & 1 & 0 \\
8 & POM & $3 / 5 / 7$ & 5.6 & 1 & 5 \\
9 & POM & $3 / 5 / 7$ & 5.6 & 1 & 10 \\
10 & Glass & $5 / 7 / 10$ & 8 & 1 & 0 \\
11 & Glass & $5 / 7 / 10$ & 8 & 1 & 2.5 \\
12 & Glass & $5 / 7 / 10$ & 8 & 1 & 5 \\
13 & Glass & $5 / 7 / 10$ & 8 & 0.8 & 0 \\
14 & Glass & $5 / 7 / 10$ & 8 & 0.8 & 2.5 \\
15 & Glass & $5 / 7 / 10$ & 8 & 0.8 & 5 \\
16 & Glass & $3 / 5 / 7$ & 5.6 & 1 & 0 \\
17 & Glass & $3 / 5 / 7$ & 5.6 & 1 & 2.5 \\
18 & Glass & $3 / 5 / 7$ & 5.6 & 1 & 5 \\
\hline
\end{tabular}

595

596 In Fig. 10 and Fig. 12, the results are presented as fraction retained over time which is 


$$
Y=Y(t)=m_{p, l} / m_{p, l, 0}
$$

597 where $m_{p, l, 0}$ is the initial mass at $t=0 \mathrm{~s}$ and $m_{p, l}$ is the remaining mass of the particles and the

598 liquid which is not in the collecting bin at time $t$. The fraction retained can also be stated per 599 particle size class $i$, if the undersized particles should be considered as different fractions in 600 the DEM simulations which is exemplarily shown in Fig. 13. This resolved fraction retained can 601 be calculated as

$$
Y_{i}=Y_{i}(t)=m_{p, l, i} / m_{p, l, i, 0}
$$

602

603

604

605

606

a

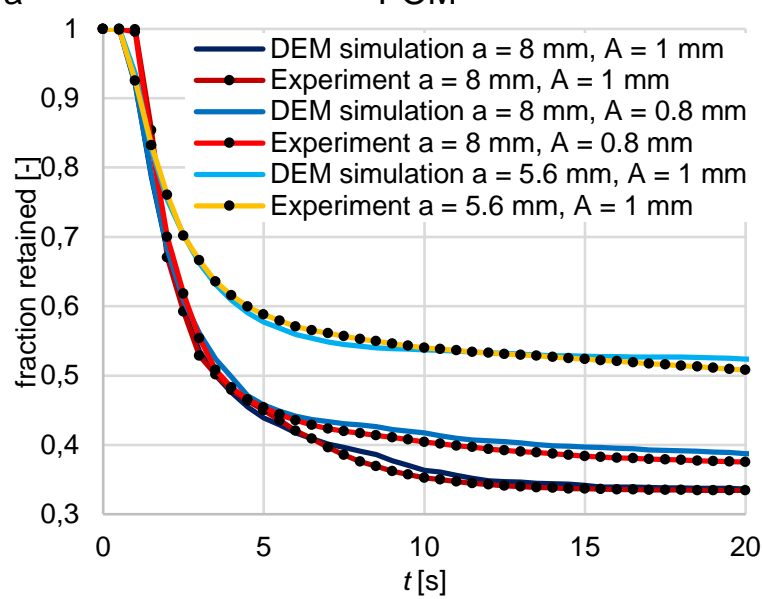
assigned to the particles. b

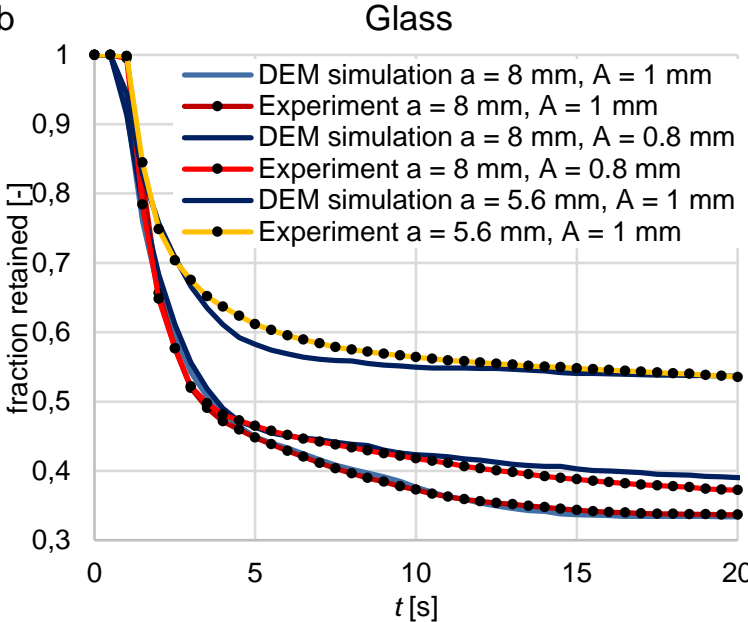

607

608

609

610

611

612

613

614

615

616

617

618

619

620

Here, $m_{p, l, i}$ and $m_{p, l, i, 0}$ are the actual and initial fractional mass of the particles plus the liquid

In the first investigations, dry material with different size classes is screened (Simulation Nos. $1,4,7,10,13,16)$ and the experimental results for the fraction retained on the screen over time are compared to the ones obtained by DEM simulations in Fig. 10.

Fig. 10: Fraction retained on the screen over time applying (a) dry POM spheres with $a=8 \mathrm{~mm}\left(d_{1 / 2 / 3}=5 / 7 / 10 \mathrm{~mm}\right)$ with $A=0.8 \mathrm{~mm}$ and $A=1 \mathrm{~mm}$ as well as $a=5.6 \mathrm{~mm}\left(d_{1 / 2 / 3}=3 / 5 / 7 \mathrm{~mm}\right)$ with $A=1 \mathrm{~mm}$ and (b) dry glass spheres with $a=8 \mathrm{~mm}$ $\left(d_{1 / 2 / 3}=5 / 7 / 10 \mathrm{~mm}\right)$ with $A=0.8 \mathrm{~mm}$ and $A=1 \mathrm{~mm}$ as well as $a=5.6 \mathrm{~mm}\left(d_{1 / 2 / 3}=3 / 5 / 7 \mathrm{~mm}\right)$ with $A=1 \mathrm{~mm}$. All results are obtained by experimental investigations (results are averaged over 15 experiments) and DEM simulations, respectively.

Besides some minor deviations, the simulation results of POM spheres fit the experimental ones very well (comp. Fig. 10a). For an aperture size of $a=8 \mathrm{~mm}\left(d_{1 / 2 / 3}=5 / 7 / 10 \mathrm{~mm}\right)$, an amplitude of $A=1 \mathrm{~mm}$ (now referred to as initial configuration, independent of $M$ ) results in a fast reduction of the fraction retained value until all particles are screened at $t \approx 15 \mathrm{~s}$. In contrast, an amplitude of $A=0.8 \mathrm{~mm}$ reduces the passing of particles after $t=5 \mathrm{~s}$ due to shorter particle throws resulting in less possibilities for the smaller particles to pass through gaps between coarse particles and the screen surface in the direction of the apertures. When an aperture size of $a=5.6 \mathrm{~mm}\left(d_{1 / 2 / 3}=3 / 5 / 7 \mathrm{~mm}\right)$ is applied, the particles pass the apertures fast in the first seconds, but after $t=2 \mathrm{~s}$ the passing is reduced and takes longer than in the initial configuration, both in experiment and DEM simulation. After the first layers of undersized 
particles have passed the apertures, the larger particles peg the apertures more intensively than in the initial configuration. Thereafter, the stratification through the coarse material to the screen surface is hindered. The results for dry glass spheres are very similar, but some larger deviations occur when applying a smaller aperture size (comp. Fig. 10b). The retardation is slightly more intensive, both in experiment and in DEM simulation. In the next investigations, small liquid amounts are added to the particles. In Fig. 11 the visualization of the liquid distribution on the particles and walls as liquid film thickness is presented in ascending intensity of blue tones at $t=3 \mathrm{~s}$. Some of the undersized particles have already passed the apertures and the remaining ones reveal thinner liquid films than the larger particles. Most of the large wall elements claim only thin liquid films.

a

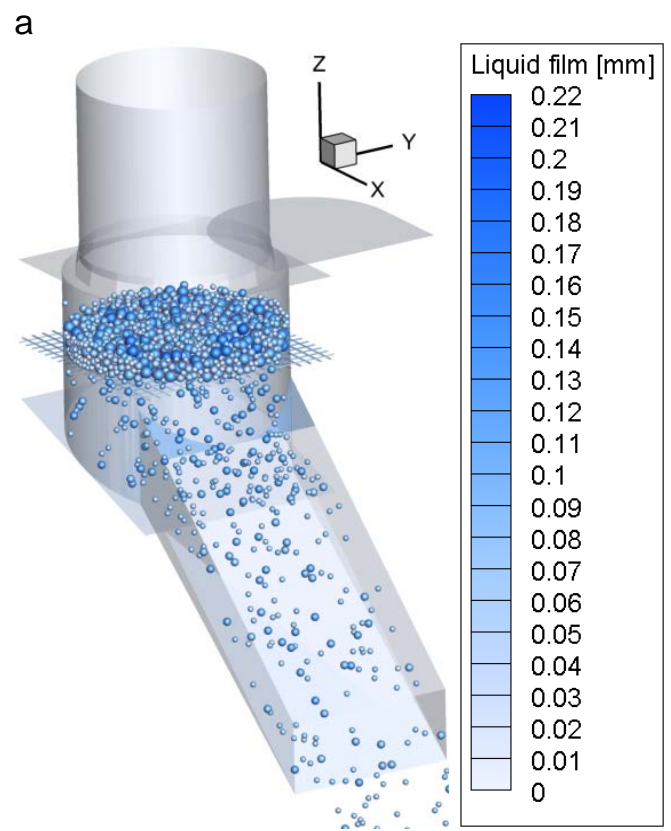

b

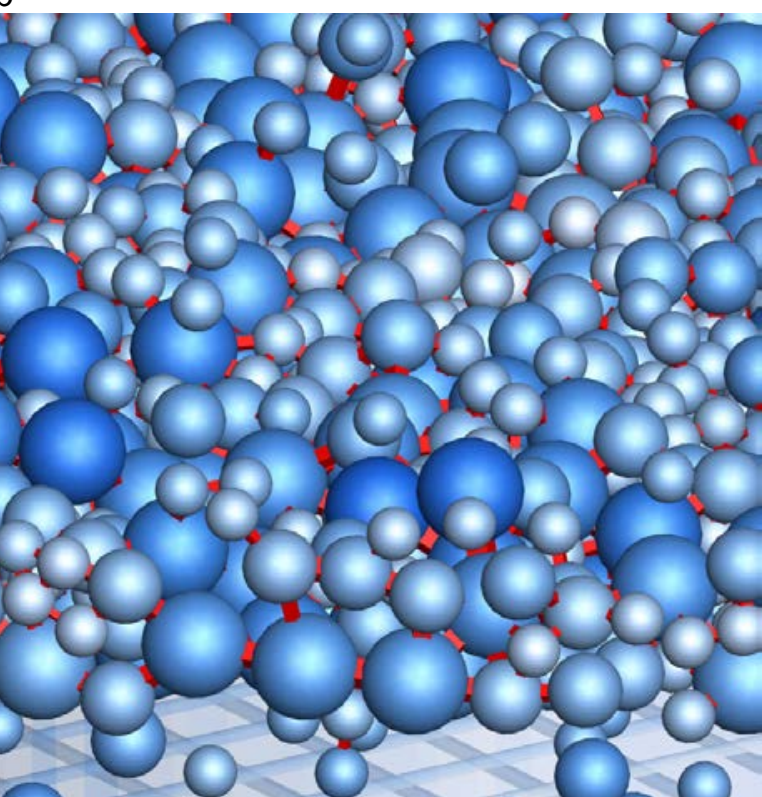

631

632

633

634

635

636

637

638

639

640

641

642

643

644

Fig. 11: Visualization of the liquid distribution on the particles and walls presented as liquid film thickness at $t=3 \mathrm{~s}$ for POM spheres $\left(a=8 \mathrm{~mm}, d_{1 / 2 / 3}=5 / 7 / 10 \mathrm{~mm}\right)$ and a liquid amount of $M=10 \%$ for (a) the whole screen apparatus and (b) zoomed in to reveal the liquid bridge volume between particles presented as cuboids.

The close-up in Fig. 11b additionally reveals the liquid bridges between the particles and their respective volumes, presented as cuboids. The cuboids always have the length $d_{l b}$, hence, a part of them is inside the spherical caps of the two connected particles. The volume of the cuboids, which is visible outside the spheres, is equal to the volume of the liquid bridge. The cuboids are stretched when one particle move away from another until the liquid bridge ruptures. The screen wires also show relatively thin liquid films.

In Fig. 12, the experimental results for the fraction retained on the screen over time for dry particles and particles under the influence of different liquid amounts are compared to the ones obtained by DEM simulations. In the initial configuration (comp. Fig. 12a), a small liquid amount $(M=5 \%)$ reduces the particle passage, whereas a larger amount $(M=10 \%)$ does not further impair it, both in experiment and simulation. The influence of the water is comparatively low 
645 due to the large contact angles and particle sizes. Therefore, the capillary force is low in 646 comparison to the weight force. In the DEM simulations, slightly more particles remain on the 647 screen between $t=2.5-10 \mathrm{~s}$, but afterwards the results fit very well.
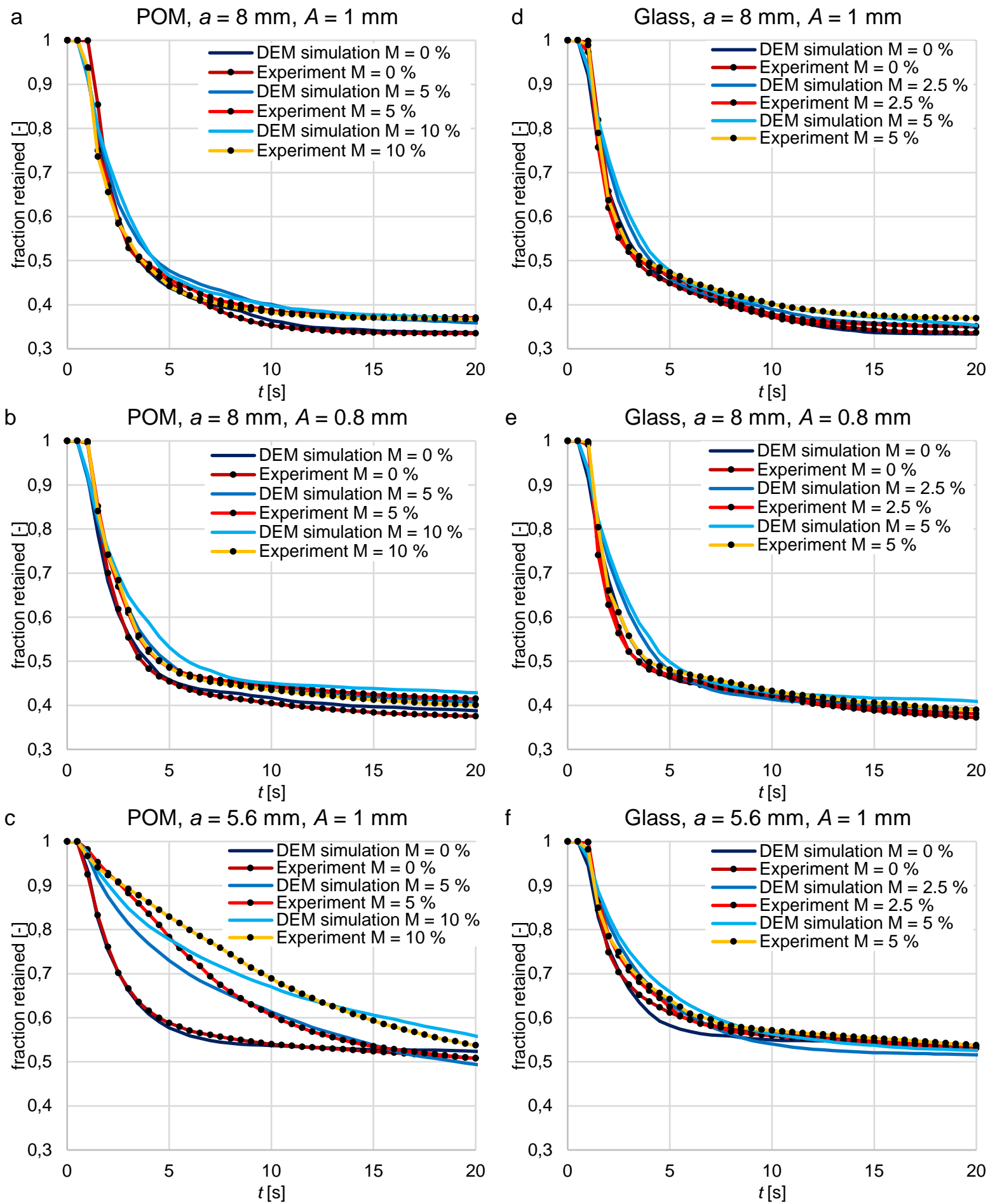

Fig. 12: Fraction retained on the screen over time applying (a,b,c) dry and wet $(M=0 / 5 / 10 \%)$ POM spheres with $a=8 \mathrm{~mm}$ $649\left(d_{1 / 2 / 3}=5 / 7 / 10 \mathrm{~mm}\right)$ with (a) $A=1 \mathrm{~mm}$ and (b) $A=0.8 \mathrm{~mm}$ as well as (c) $a=5.6 \mathrm{~mm}\left(d_{1 / 2 / 3}=3 / 5 / 7 \mathrm{~mm}\right)$ with $A=1 \mathrm{~mm}$ as well as 650 (d,e,f) dry and wet $(M=0 / 2.5 / 5 \%)$ glass spheres with $a=8 \mathrm{~mm}\left(d_{1 / 2 / 3}=5 / 7 / 10 \mathrm{~mm}\right)$ with (d) $A=1 \mathrm{~mm}$ and $(\mathrm{e}) A=0.8 \mathrm{~mm}$ as well 651 as $(\mathrm{f}) a=5.6 \mathrm{~mm}\left(d_{1 / 2 / 3}=3 / 5 / 7 \mathrm{~mm}\right)$ with $A=1 \mathrm{~mm}$. All results are obtained by experimental investigations (results are averaged over 15 experiments) and DEM simulations, respectively. 
653 When an amplitude of $A=0.8 \mathrm{~mm}$ is applied (comp. Fig. 12b), the fraction retained value is 654 higher if water is added to the particles. The experimental and numerical results for a lower 655 amount of water $(M=5 \%)$ fit very well. However, in the DEM simulations, the fraction retained 656 for a larger amount of water $(M=10 \%)$ is slightly overpredicted. The influence of liquid is more 657 pronounced for the configuration with smaller particle diameters $d_{1 / 2 / 3}=3 / 5 / 7 \mathrm{~mm}$ (comp. 658 Fig. 12c). Until $t=20 \mathrm{~s}$ the particle passage is reduced for $M=5 \%$ and even more retarded 659 for $M=10 \%$. With smaller particle sizes applied in this investigation, the adhesive forces 660 become larger relative to the weight force, which is relatively small due to the low density of 661 POM. Due to the pegging of particles in the dry case, the fraction retained is similar after $662 t=20 \mathrm{~s}$ for $M=0 \%$ and $M=5 \%$ and only slightly larger for $M=10 \%$. The DEM simulations 663 show the same trends but reveal some deviations between $t=1-10 \mathrm{~s}$.

664 Applying the initial configuration with glass spheres as material (comp. Fig. 12d), a larger 665 amount of water increases the experimentally and numerically obtained fraction retained. However, the influence of the water is relatively low due to the large particle size, density and the related masses. The simulation results under the influence of liquid both reveal some deviations between $t=2-5 \mathrm{~s}$. Afterwards, the results fit very well. The same trends are recognizable for an amplitude of $A=0.8 \mathrm{~mm}$ (comp. Fig. 12e). Here, all the results are closer 670 together.

671 The results for the glass spheres with smaller particle diameters of $d_{1 / 2 / 3}=3 / 5 / 7 \mathrm{~mm}$ (comp. 672 Fig. 12f) are very different from those obtained with POM spheres. Particularly, the 673 experimental results lie close to each other with slightly larger values if more water is added to 674 the particles. Due to the larger density of glass spheres, the influence of the weight force 675 compared to the capillary force is more intense than for POM. The simulation results reveal a 676 bit more differences and slightly overpredict the fraction retained until $t \approx 7 \mathrm{~s}$ and underpredict 677 it afterwards. Due to the pegging of the dry particles, fewer particles remain on the screen at $678 t=20 \mathrm{~s}$ if water is added before the screening process. Overall, the simulation results fit the 679 experimental ones quite good. The most deviations for the configurations with the larger 680 diameters $d_{1 / 2 / 3}=5 / 7 / 10 \mathrm{~mm}$ are the result of overpredictions while in the other configurations 681 under- and overpredictions occur.

682 The simulation results of the fraction retained resolved for the two undersized fractions (near 683 mesh and small) are shown in Fig. 13 for various liquid amounts. In most of the configurations, 684 the smaller particles with the diameter $d_{3}$ pass the apertures faster than the near mesh particles 685 with the diameter $d_{2}$. Besides one exception, this is also valid for the initial configuration. The 686 smaller particles $\left(d_{3}\right)$ at a liquid amount of $M=10 \%$ need more time to pass the apertures at 
687 the beginning (until $t=7 \mathrm{~s}$ ), but then show expected results. Here, the water seems to have a 688 large influence on the small particles $\left(d_{3}\right)$.
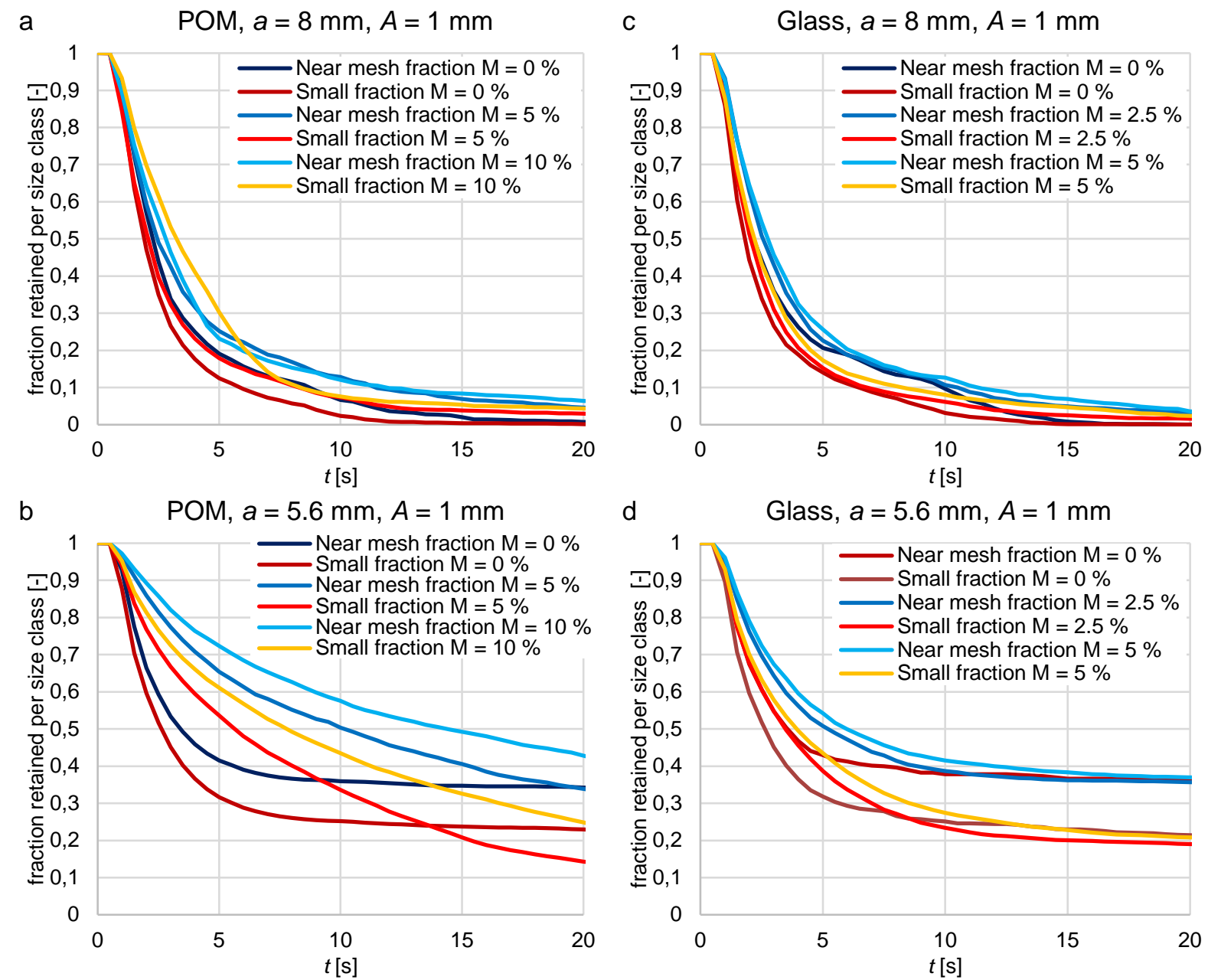

689

690

691

692

693

694

695

696

697

698

699

700

701

702

703

704

Fig. 13: Fraction retained on the screen over time presented for the small $\left(d_{3}\right)$ and near mesh sized particle fractions $\left(d_{2}\right)$ applying dry and wet $(M=0 / 5 / 10 \%)$ POM spheres with (a) $a=8 \mathrm{~mm}\left(d_{1 / 2 / 3}=5 / 7 / 10 \mathrm{~mm}\right)$ and (b) $a=5.6 \mathrm{~mm}\left(d_{1 / 2 / 3}=3 / 5 / 7 \mathrm{~mm}\right)$ as well as dry and wet $(M=0 / 2.5 / 5 \%)$ glass spheres with (c) $a=8 \mathrm{~mm}\left(d_{1 / 2 / 3}=5 / 7 / 10 \mathrm{~mm}\right)$ and $(\mathrm{d}) a=5.6 \mathrm{~mm}\left(d_{1 / 2 / 3}=3 / 5 / 7 \mathrm{~mm}\right)$ all with $A=1 \mathrm{~mm}$. All results are obtained by DEM simulations.

In the configuration with an aperture size of $a=5.6 \mathrm{~mm}$ (comp. Fig. 13b), the particles are more influenced by the water, but pass the apertures as estimated in terms of small and near mesh sized particles. Here, it is even more obvious, that after a while, the small and near mesh sized dry particles are hindered from passing the apertures. In both configurations where an amount of water is added to the particles, the passage is slower in the beginning of the screening process, but it is not hindered and for $M=5 \%$ even less particles remain on the screen. If glass spheres are applied in the initial configuration (comp. Fig. 13c), the resolved fraction retained values are as expected before. Interesting is that the small particles $\left(d_{3}\right)$ under the influence of a liquid amount of $M=5 \%$ temporarily pass the apertures even slower than the dry near mesh sized particles $\left(d_{2}\right)$. Besides the peculiarities mentioned about the not resolved fraction retained (comp. Fig. 12f), the configuration with small glass spheres (comp. Fig. 13d) shows expected results. 


\subsection{Liquid distribution in experiments and DEM simulations}

706

707

708

709

710

711

712

713

714

715

716

717

718

In order to find out more about the reasons for the occurred deviations, a closer look should be taken at the liquid distribution. Hence, in Fig. 14 the liquid distribution at the end of the screening process is compared between experimental measurements and results obtained by DEM simulations for POM (Fig. 14a) and glass spheres (Fig. 14b). Note that the residuals (light blue contour, comp. Fig. 14c) are due to mixing, transferring, evaporation and slots in the apparatus. This amount is subtracted from the liquid amount before the simulation. The other divisions (comp. Fig. 14c) are the feed bin, the side walls and the outlet (red contour), the coarse material and the screen wires plus the side walls of the screen (purple contour) and the fine particles plus the collecting bin (green contour). Note that the liquid of the currently existing liquid bridges is assigned by means of the transfer ratio (comp. section 4) to the particles or walls for the evaluations concerning the liquid distribution. When applying POM spheres in the DEM simulations (comp. Fig. 14a), the amount of water on the screen and on the coarse material as well as on the other wall elements is larger than in the experiments.

a POM, $a=8 \mathrm{~mm}, A=1 \mathrm{~mm}, M=5 \%$ Experiment (\%)

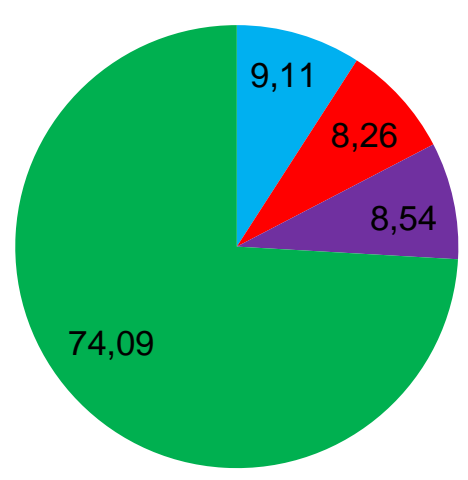

b Glass, $a=8 \mathrm{~mm}, A=1 \mathrm{~mm}, M=2.5 \%$ Experiment (\%)

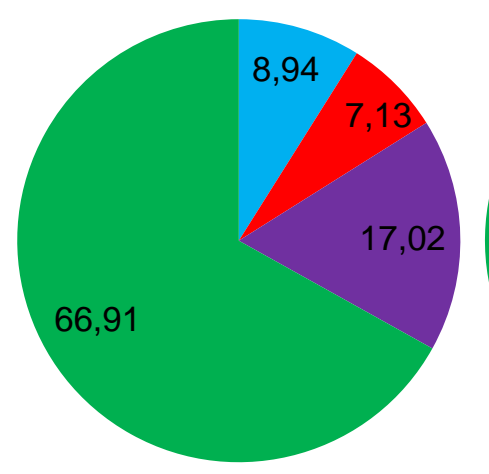

Simulation (\%)

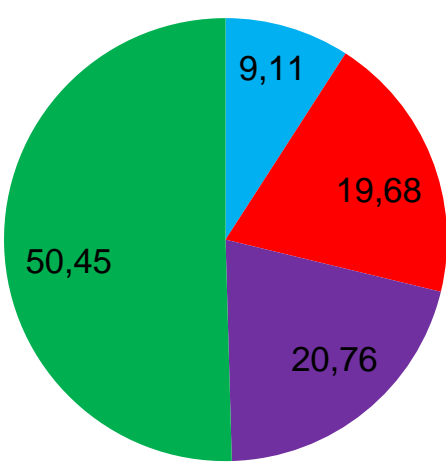

Simulation (\%)
C

Residuals

- Feed bin, side walls, outlet

- Coarse particles, screen wires, screen side walls

Fine particles, collecting bin

Fig. 14: Liquid distribution of the screening process at $t=20 \mathrm{~s}$ compared between experiments and simulations for (a) POM 720 spheres with $M=5 \%$ and (b) glass spheres with $M=2.5 \%$ both with $a=8 \mathrm{~mm}\left(d_{1 / 2 / 3}=5 / 7 / 10 \mathrm{~mm}\right)$ and $A=1 \mathrm{~mm}$. (c) Visualization 721 of the divisions of the screening apparatus and the applied material covered by liquid. 
The liquid amount on the coarse particles and on the screen is also overpredicted in case of glass spheres (comp. Fig. 14b), but the other wall elements hold about the same amount of water in the experiments and the DEM simulations. Due to the large contact angle of POM, the liquid amount on the particles is lower in comparison to the glass spheres. When a liquid bridge between a wall and a POM sphere ruptures, the wall element takes a comparatively larger amount of liquid than after the rupture of a bridge with a glass sphere. After the liquid bridge between two glass spheres ruptures, relatively more water is assigned to the larger sphere in comparison to the configuration with POM spheres for a dimensionless liquid volume of $V_{l b}^{*}<0.4$, which is valid in the investigation here (comp. Fig. 7). Due to these reasons, the proportion of water on the coarse glass spheres is larger than on the coarse POM spheres.

The main reason for the underprediction of water on the fine particles and in the collecting bin is that the water in the simulations persists only on particles, walls and in liquid bridges. In contrast, the water in the experiments can also be separated from particles and walls in free motion. The liquid separation from particles due to vibrating dewatering is not realized in the DEM simulations until now and is required to be addressed in further investigations. First studies with the same configurations but with smaller apertures $\left(a<d_{3}\right)$ reveal that the amount of water which pass through the outlet is approximately equal to the amount of water that is overpredicted on the coarse particles (and wall elements in case of POM) in the simulations. By considering this, the prediction of the amount of water, which adheres to the fine particles, might be correct, but the water accumulating in the collecting bin is not taken into account in the DEM simulations.

In Fig. 15, the liquid distribution over time during the screening process in the DEM simulations is shown. Here, it is possible to consider the various parts of the screen apparatus (all wall elements) separately (screen wires, feed bin, outlet, side walls) and only the liquid on and between the particles remaining on top of the screen is referred to as "coarse material". Note that the liquid between two particles or particles and walls at time $t$ is split up on the respective particle or wall like when the bridge would rupture at that point in time $t$ (comp. section 4). The liquid on the particles that passed the outlet is referred to as "fines" in Fig. 15. To avoid confusions and redundancies, the division of the coarse material and fines into the particle fractions instead of the division of the screen apparatus is only shown for one case with glass spheres in Fig. 15c.

At $t=0 \mathrm{~s}$, the water persists only on the particles above the screen (coarse material) in all simulations. The same liquid film thickness $P_{l f}$ is applied for each particle (comp. section 5.1). Directly in the first time step, the first liquid bridges form out between particles as well as particles and walls. The amount of liquid of the coarse material decreases continuously, while 
the amount of liquid on the wall elements and particularly of the fines increases. Latter is not only due to the transfer of water between particles, but also due to small and near mesh particles passing through the apertures whereby they turn from coarse material to the fines. At the end of a screening process under investigated configurations (not necessarily $t=20 \mathrm{~s}$ ), probably more liquid is assigned to the fines than to the coarse material which is already the case for the configurations shown in Figs. 15a,c. The increase of the amount of water on the wall elements is more pronounced in the simulations with POM spheres due to their lower wettability or rather their high contact angle. In Fig. 15a, the liquid amount on the screen apparatus is nearly equal to the liquid amount on the coarse particles at $t=20 \mathrm{~s}$ and still gets closer. At this time, nearly all undersized particles have passed the apertures. Therefore, the change in the liquid amount is mainly due to the transfer between coarse particles and wall elements.

a

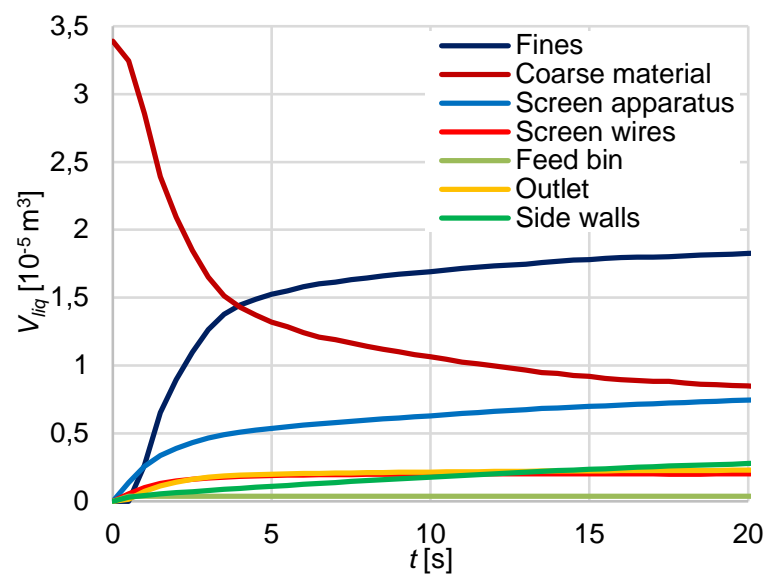

b $\quad$ POM, $a=5.6 \mathrm{~mm}, A=1 \mathrm{~mm}, M=10 \%$

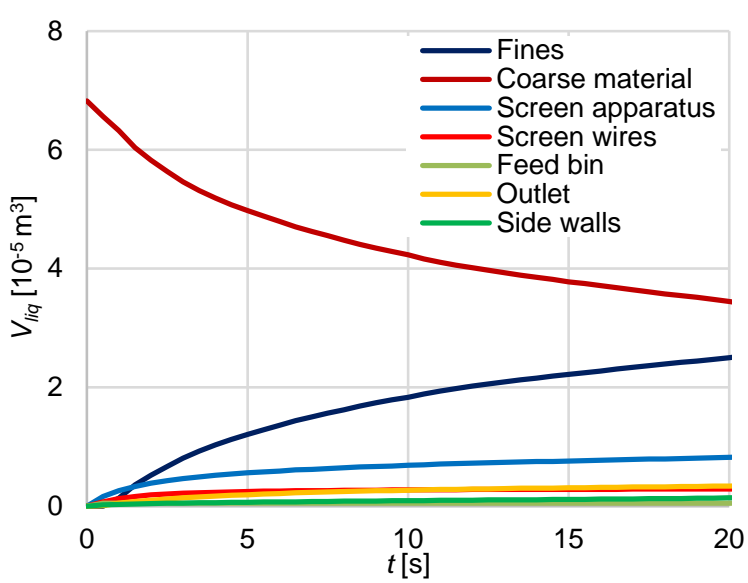

c Glass, $a=8 \mathrm{~mm}, A=1 \mathrm{~mm}, M=2.5 \%$

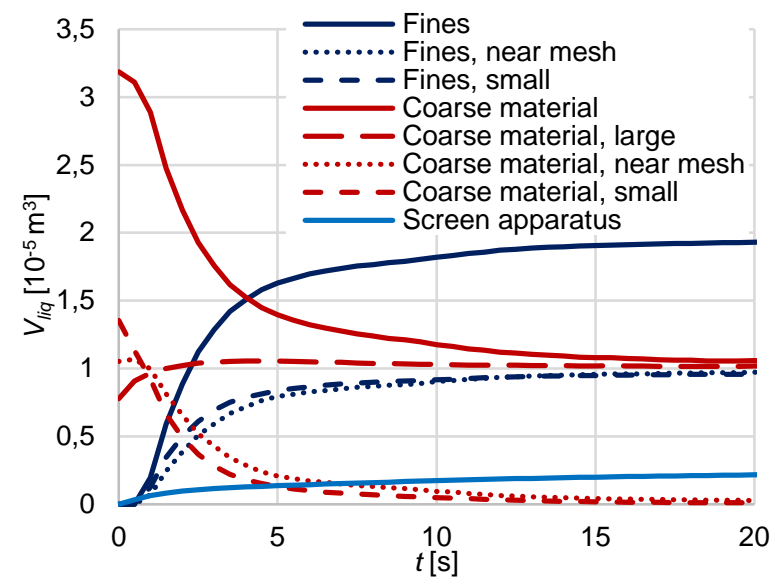

Glass, $a=5.6 \mathrm{~mm}, A=1 \mathrm{~mm}, M=5 \%$

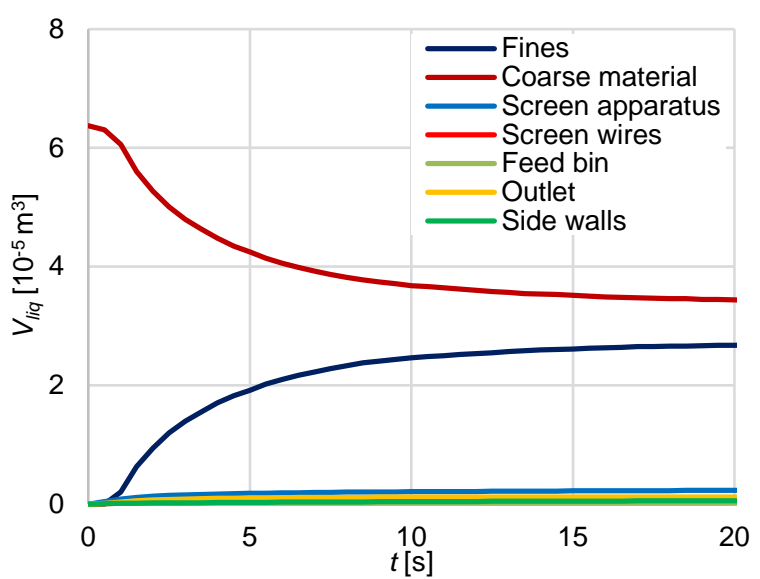

Fig. 15: Liquid distribution as liquid volume $V_{\text {liq }}$ over time $t$ during the screening process for POM spheres with $M=5 \%$ and (a) $a=8 \mathrm{~mm}\left(d_{1 / 2 / 3}=5 / 7 / 10 \mathrm{~mm}\right)$ as well as $(\mathrm{b}) a=5.6 \mathrm{~mm}\left(d_{1 / 2 / 3}=3 / 5 / 7 \mathrm{~mm}\right)$ and glass spheres with $M=2.5 \%$ and (c) $a=8 \mathrm{~mm}$ $\left(d_{1 / 2 / 3}=5 / 7 / 10 \mathrm{~mm}\right)$ as well as (d) $a=5.6 \mathrm{~mm}\left(d_{1 / 2 / 3}=3 / 5 / 7 \mathrm{~mm}\right)$ all with $A=1 \mathrm{~mm}$. All results are obtained by DEM simulations.

\begin{abstract}
In contrast to POM spheres, the liquid distribution in case of glass spheres (Figs. 15c,d) nearly
\end{abstract} reaches an equilibrium when the majority of the undersized particles are screened. In Fig. 15c 
774 it is also visible, that for the coarse material the amount of liquid on the near mesh and

775 particularly on the large particles increases at the beginning of the screening process, whereas

776 the amount of liquid on the small particles decreases at the same time. Both is mainly due to 777 the transfer ratio between different sized spheres. Additionally, Fig. 15c provides the 778 information, that slightly more water gets through the outlet on small than on near mesh 779 particles. A larger amount of small particles passes the apertures, but each near mesh particle 780 hold more water. In case of an aperture size of $a=5.6 \mathrm{~mm}$ for both materials, some of the 781 smaller particles are still $(t=20 \mathrm{~s})$ on top of the screen resulting in a larger amount of water 782 assigned to the coarse particles compared to the fines.

\section{6. Conclusions}

784 In this work, capillary and viscous force models for liquid bridge contacts as well as the 785 formation and rupture of liquid bridges have been applied in DEM simulations. The 786 implemented force models were successfully validated against data from literature and 787 additionally, the capillary force models were compared with each other. The most appropriate 788 capillary model, here the one by Rabinovich et al. [16], was applied in subsequent DEM 789 simulations. In addition, the normal and tangential viscous force models by Pitois et al. [44] 790 and Goldman et al. [45], respectively, were chosen. For the rupture distance, the model by 791 Willett et al. [18] extended for dynamic behavior by Pitois et al. [48] was selected. The 792 implemented formation and rupture process of the liquid bridge between two spheres is based 793 on the geometrical considerations of the model by Shi and McCarthy [43]. For the formation 794 and rupture between a particle and a wall, similar geometrical considerations were made and 795 used for the DEM simulations. The required contact angles of glass $\left(\theta_{i}=15^{\circ}\right)$ and POM $796\left(\theta_{i}=40^{\circ}\right)$ spheres as well as of steel $\left(\theta_{w}=45^{\circ}\right)$ and treated PVC $\left(\theta_{w}=50^{\circ}\right)$ with water and 797 respective transfer ratios were obtained and implemented in the DEM by generating a look-up 798 table. Furthermore, a method to detect existing liquid bridge contacts at large distances over 799 different cells and to transfer liquid bridge contact information and history over process 800 boundaries has been introduced.

801 Based on this, experimental and numerical batch screening has been performed. The applied 802 DEM code is capable to simulate dry screening processes very well with only minor deviations. 803 For the applied screen apparatus, a set amplitude of $A=1 \mathrm{~mm}$ and larger particles $804\left(d_{1 / 2 / 3}=5 / 7 / 10 \mathrm{~mm}\right)$ accompanied by a larger aperture size $(a=8 \mathrm{~mm})$ reveal a slightly lower 805 fraction retained than an amplitude of $A=0.8 \mathrm{~mm}$ and a much lower fraction retained than 806 smaller particles $\left(d_{1 / 2 / 3}=3 / 5 / 7 \mathrm{~mm}\right)$ accompanied by a smaller aperture size $(a=5.6 \mathrm{~mm})$. 
An addition of water slightly reduces the particle passage in most of the applied configurations, whereas blocking of apertures by particles can be reduced in some cases. The extended DEM code is able to simulate screening under the influence of a slight amount of water $(M \leq 10 \%)$. The results fit the experimental ones, while revealing slightly more deviations than for the dry configuration. Small particles mostly pass the apertures faster than near mesh sized particles independent of the amount of water. In some configurations, more small particles under the influence of moisture remain on the screen than dry near mesh sized particles.

814 The amount of water on particles, which are able to pass the apertures, during and at the end 815 of the screening process, is underpredicted. If POM spheres are applied, too much water is 816 predicted on the walls and coarse particles. In the case of glass spheres, the deviations are 817 lower, due to their good wettability. Only the coarse material reveals to have more water 818 attached in the simulation than in the experiment. Approximately the same amount of water is associated to the fine particles in the experiment. The main reason for these deviations is that the liquid in the DEM simulations only persists on particles, walls and in liquid bridges, whereas in reality, the liquid is also separated from particles and walls in free motion. The liquid bridge models are not able to take this in account and no reliable and directly applicable correlations are available. The liquid separation due to vibrating dewatering will be addressed in further studies concerning wet screening applications. For this purpose, coupled discrete element simulations with methods used for simulating the dynamics of continua like the SPH can be applied (comp. e.g. [77]). By utilizing the SPH, the fluid flow and the interaction between fluid and solid as well as the local liquid amounts can be obtained. As long as the local liquid amount is small enough, the liquid bridge models are still applicable. Additionally, resolved liquid distribution models (comp. [24]) can be used in the DEM to account for partial wetting, particularly if large contact angles are applied.

831 Furthermore, in order to meet requirements for real particle systems such as encountered in 832 industrial applications, the implemented liquid bridge models will be extended to be applied for 833 non-spherical shaped particles under moist conditions in the DEM in the future. Therefore, the 834 liquid bridge force calculation will be realized analogously and the liquid contact detection rules 835 will be combined with already implemented routines for the detection of non-spherical particles. 836 However, new methods for the liquid distribution on the individual spheres will be required. The 837 data obtained from the DEM simulations in the investigation here will be used for extending 838 phenomenological process models to represent screening processes under moist conditions 839 in consecutive studies. 


\section{Acknowledgements}

841 The authors gratefully acknowledge the support by DFG within project SPP 1679 through grant 842 number KR3446/7-2 and KR3446/7-3. The original form of the DEM-code "DEM-Calc" applied 843 is based on a development of LEAT, Ruhr-Universität Bochum, Germany. The code "DEM844 Calc" has then been continuously extended both at Ruhr-Universität Bochum and Technische Universität Berlin, Germany. We thank all who have contributed.

\section{Compliance with ethical standards}

The authors declare that there is no conflict of interest related to this manuscript.

848

849

850

851

852

853

854

855

856

857

858

859

860

861

862

863

864

865

866

867

868

869

870

871

872

873

874

875

876

877

878

\section{References}

[1] Liu, K.: Some factors affecting sieving performance and efficiency. Powder Technol. 193, 208-213 (2009)

[2] Grozubinsky, V., Sultanovitch, E., Lin, I.J.: Efficiency of solid particle screening as a function of screen slot size, particle size, and duration of screening - The theoretical approach. Int. J. Miner. Process. 52, 261-272 (1998)

[3] Guerreiro, F.S., Gedraite, R., Ataíde, C.H.: Residual moisture content and separation efficiency optimization in pilot-scale vibrating screen. Powder Technol. 287, 301-307 (2016)

[4] Keller, K.: Schwingentwässerung von körnigen Produkten. Dissertation, Universität Karlsruhe, VDI Verlag, Karlsruhe (1997)

[5] Govender, A., van Dyk, J.C.: Effect of wet screening on particle size distribution and coal properties. Fuel 82, 2231-2237 (2003)

[6] Robertson, J., Thomas, C.J., Caddy, B., Lewis, A.J.M.: Particle Size Analysis of Soils A Comparison of Dry and Wet Sieving Techniques. Forensic Sci. Int. 24, 209-217 (1984)

[7] Cundall, P.A., Strack, O.D.L.: A discrete numerical model for granular assemblies. Geotechnique 29, 47-65 (1979)

[8] Cleary, P.W., Sinnott, M.D., Morrison, R.D.: Separation performance of double deck banana screens - Part 1: Flow and separation for different accelerations. Miner. Eng. 22, 1218-1229 (2009)

[9] Cleary, P.W., Sinnott, M.D., Morrison, R.D.: Separation performance of double deck banana screens - Part 2: Quantitative predictions. Miner. Eng. 22, 1230-1244 (2009)

[10] Delaney, G.W., Cleary, P.W., Hilden, M., Morrison, R.D.: Testing the validity of the spherical DEM model in simulating real granular screening processes. Chem. Eng. Sci. 68, 215-226 (2012)

[11] Kruggel-Emden, H., Elskamp, F.: Modeling of Screening Processes with the Discrete Element Method Involving Non-Spherical Particles. Chem. Eng. Technol. 37, 847-856 (2014)

[12] Yoshida, Y., Ishikawa, S., Shimosaka, A., Shirakawa, Y., Hidaka, J.: Estimation Equation for Sieving Rate Based on the Model for Undersized Particles Passing through 
[13] Dong, K.J., Yu, A.B.: Numerical simulation of the particle flow and sieving behaviour on sieve bend/low head screen combination. Miner. Eng. 31, 2-9 (2012)

882

[14] Fernandez, J.W., Cleary, P.W., Sinnott, M.D., Morrison, R.D.: Using SPH one-way coupled to DEM to model wet industrial banana screens. Miner. Eng. 24, 741-753 (2011)

885

886

887

888

889

890

891

892

893

894

895

896

897

898

899

900

901

902

903

904

905

906

907

908

909

910

911

912

913

914

915

916

917

918

919

920

921

[15] Zhu, H.P., Zhou, Z.Y., Yang, R.Y., Yu, A.B.: Discrete particle simulation of particulate systems: Theoretical developments. Chem. Eng. Sci. 62, 3378-3396 (2007)

[16] Rabinovich, Y.I., Esayanur, M.S., Moudgil, B.M.: Capillary forces between two spheres with a fixed volume liquid bridge: Theory and experiment. Langmuir 21, 10992-10997 (2005)

[17] Lambert, P., Chau, A., Delchambre, A.: Comparison between Two Capillary Forces Models. Langmuir 24, 3157-3163 (2008)

[18] Willett, C.D., Adams, M.J., Johnson, S.A., Seville, J.P.K.: Capillary Bridges between Two Spherical Bodies. Langmuir 16, 9396-9405 (2000)

[19] Weigert, T., Ripperger, S.: Calculation of the Liquid Bridge Volume and Bulk Saturation from the Half-filling Angle. Part. Part. Syst. Charact. 16, 238-242 (1999)

[20] Israelachvili, J.N.: Intermolecular and Surface Forces. 3rd ed.Academic Press, London (2011)

[21] Radl, S., Kalvoda, E., Glasser, B.J., Khinast, J.G.: Mixing characteristics of wet granular matter in a bladed mixer. Powder Technol. 200, 171-189 (2010)

[22] Tsunazawa, Y., Fujihashi, D., Fukui, S., Sakai, M., Tokoro, C.: Contact force model including the liquid-bridge force for wet-particle simulation using the discrete element method. Adv. Powder Technol. 27, 652-660 (2016)

[23] Liu, P.Y., Yang, R.Y., Yu, A.B.: DEM study of the transverse mixing of wet particles in rotating drums. Chem. Eng. Sci. 86, 99-107 (2012)

[24] Washino, K., Miyazaki, K., Tsuji, T., Tanaka, T.: A new contact liquid dispersion model for discrete particle simulation. Chem. Eng. Res. Des. 110, 123-130 (2016)

[25] Washino, K., Chan, E.L., Miyazaki, K., Tsuji, T., Tanaka, T.: Time step criteria in DEM simulation of wet particles in viscosity dominant systems. Powder Technol. 302, 100107 (2016)

[26] Song, C., Liu, D., Ma, J., Chen, X.: CFD-DEM simulation of flow pattern and particle velocity in a fluidized bed with wet particles. Powder Technol. 314, 346-354 (2017)

[27] Fu, J., Adams, M.J., Reynolds, G.K., Salman, A.D., Hounslow, M.J.: Impact deformation and rebound of wet granules. Powder Technol. 140, 248-257 (2004)

[28] Antonyuk, S., Heinrich, S., Deen, N., Kuipers, H.: Influence of liquid layers on energy absorption during particle impact. Particuology 7, 245-259 (2009)

[29] Antonyuk, S., Dosta, M., Heinrich, S.: Numerical estimation of the restitution coefficient for dry and wet agglomerates. AIP Conf. Proc. 1542, 951-954 (2013)

[30] Crüger, B., Salikov, V., Heinrich, S., Antonyuk, S., Sutkar, V.S., Deen, N.G., Kuipers, J.A.M.: Coefficient of restitution for particles impacting on wet surfaces: An improved experimental approach. Particuology 25, 1-9 (2016)

[31] Crüger, B., Heinrich, S., Antonyuk, S., Deen, N.G., Kuipers, J.A.M.: Experimental study 
of oblique impact of particles on wet surfaces. Chem. Eng. Res. Des. 110, 209-219 (2016)

[32] Sutkar, V.S., Deen, N.G., Padding, J.T., Kuipers, J.A.M., Salikov, V., Crüger, B., Antonyuk, S., Heinrich, S.: A Novel Approach to Determine Wet Restitution Coefficients Through a Unified Correlation and Energy Analysis. AIChE J. 61, 769-779 (2015)

[33] van Buijtenen, M.S., Deen, N.G., Heinrich, S., Antonyuk, S., Kuipers, J.A.M.: A discrete element study of wet particle-particle interaction during granulation in a spout fluidized bed. Can. J. Chem. Eng. 87, 308-317 (2009)

[34] Kralchevsky, P.A., Nagayama, K.: Capillary interactions between particles bound to interfaces, liquid films and biomembranes. Adv. Colloid Interface Sci. 85, 145-192 (2000)

[35] Gabrieli, F., Lambert, P., Cola, S., Calvetti, F.: Micromechanical modelling of erosion due to evaporation in a partially wet granular slope. Int. J. Numer. Anal. Methods Geomech. 36, 918-943 (2012)

[36] Lian, G., Thornton, C., Adams, M.J.: A Theoretical Study of the Liquid Bridge Forces between Two Rigid Spherical Bodies. J. Colloid Interface Sci. 161, 138-147 (1993)

[37] Fisher, R.A.: On the capillary forces in an ideal soil; correction of formulae given by W. B. Haines. J. Agric. Sci. 16, 492-505 (1926)

[38] Soulié, F., Cherblanc, F., El Youssoufi, M.S., Saix, C.: Influence of liquid bridges on the mechanical behaviour of polydisperse granular materials. Int. J. Numer. Anal. Methods Geomech. 30, 213-228 (2006)

[39] Richefeu, V., Youssoufi, M.S. El, Peyroux, R., Radja, F.: A model of capillary cohesion for numerical simulations of 3D polydisperse granular media. Int. J. Numer. Anal. Methods Geomech. 32, 1365-1383 (2008)

[40] Gladkyy, A., Schwarze, R.: Comparison of different capillary bridge models for application in the discrete element method. Granul. Matter 16, 911-920 (2014)

[41] Lian, G., Seville, J.: The capillary bridge between two spheres: New closed-form equations in a two century old problem. Adv. Colloid Interface Sci. 227, 53-62 (2016)

[42] Adams, M.J., Perchard, V.: The Cohesive Forces Between Particles with Interstitial Liquid. Inst. Chem. Eng. Symp. Ser. 91, 147-160 (1985)

[43] Shi, D., McCarthy, J.J.: Numerical simulation of liquid transfer between particles. Powder Technol. 184, 64-75 (2008)

[44] Pitois, O., Moucheront, P., Chateau, X.: Liquid Bridge between Two Moving Spheres: An Experimental Study of Viscosity Effects. J. Colloid Interface Sci. 231, 26-31 (2000)

[45] Goldman, A.J., Cox, R.G., Brenner, H.: Slow viscous motion of a sphere parall to a plane wall-I Motion through a quiescent fluid. Chem. Eng. Sci. 22, 653-660 (1967)

[46] Song, Y., Turton, R.: Study of the effect of liquid bridges on the dynamic behavior of two colliding tablets using DEM. Powder Technol. 178, 99-108 (2007)

[47] Nase, S.T., Vargas, W.L., Abatan, A.A., McCarthy, J.J.: Discrete characterization tools for cohesive granular material. Powder Technol. 116, 214-223 (2001)

[48] Pitois, O., Moucheront, P., Chateau, X.: Rupture energy of a pendular liquid bridge. Eur. Phys. J. B 23, 79-86 (2001)

[49] Pepin, X., Rossetti, D., Iveson, S.M., Simons, S.J.R.: Modeling the Evolution and Rupture of Pendular Liquid Bridges in the Presence of Large Wetting Hysteresis. J. 
[50] Schmelzle, S., Nirschl, H.: DEM simulations: mixing of dry and wet granular material with different contact angles. Granul. Matter 20:19, (2018)

969

970

971

972

973

974

975

976

977

978

979

980

981

982

983

984

985

986

987

988

989

990

991

992

993

994

995

996

997

998

999

1000

1001

1002

1003

1004

1005

1006

1007

1008

1009

[51] Wu, M., Radl, S., Khinast, J.G.: A Model to Predict Liquid Bridge Formation Between Wet Particles Based on Direct Numerical Simulations. AIChE J. 62, 1877-1897 (2016)

[52] Scholtès, L., Chareyre, B., Nicot, F., Darve, F.: Discrete modelling of capillary mechanisms in multi-phase granular media. Comput. Model. Eng. Sci. 52, 297-318 (2009)

[53] Melnikov, K., Mani, R., Wittel, F.K., Thielmann, M., Herrmann, H.J.: Grain scale modeling of arbitrary fluid saturation in random packings. Phys. Rev. E 92, 022206 (2015)

[54] Wang, J., Gallo, E., François, B., Gabrieli, F., Lambert, P.: Capillary force and rupture of funicular liquid bridges between three spherical bodies. Powder Technol. 305, 89-98 (2017)

[55] Yang, S.C., Hsiau, S.S.: The simulation of powders with liquid bridges in a 2D vibrated bed. Chem. Eng. Sci. 56, 6837-6849 (2001)

[56] Yang, R.Y., Zou, R.P., Yu, A.B.: Numerical study of the packing of wet coarse uniform spheres. AIChE J. 49, 1656-1666 (2003)

[57] Rognon, P.G., Roux, J.N., Wolf, D., Naaïm, M., Chevoir, F.: Rheophysics of cohesive granular materials. Europhys. Lett. 74, 644-650 (2006)

[58] Khamseh, S., Roux, J.-N., Chevoir, F.: Flow of wet granular materials: a numerical study. Phys. Rev. E 92, 022201 (2015)

[59] Heine, M., Antonyuk, S., Fries, L., Niederreiter, G., Heinrich, S., Palzer, S.: Modeling of the spray zone for particle wetting in a fluidized bed. Chemie Ing. Tech. 85, 280-289 (2013)

[60] Lim, E.W.C.: Density segregation of dry and wet granular mixtures in vibrated beds. Adv. Powder Technol. 27, 2478-2488 (2016)

[61] He, Y., Peng, W., Tang, T., Yan, S., Zhao, Y.: DEM numerical simulation of wet cohesive particles in a spout fluid bed. Adv. Powder Technol. 27, 93-104 (2016)

[62] Horabik, J., Molenda, M.: Parameters and contact models for DEM simulations of agricultural granular materials: A review. Biosyst. Eng. 147, 206-225 (2016)

[63] Alonso-Marroquín, F., Ramírez-Gómez, Á., González-Montellano, C., Balaam, N., Hanaor, D.A.H., Flores-Johnson, E.A., Gan, Y., Chen, S., Shen, L.: Experimental and numerical determination of mechanical properties of polygonal wood particles and their flow analysis in silos. Granul. Matter 15, 811-826 (2013)

[64] Barrios, G.K.P., de Carvalho, R.M., Kwade, A., Tavares, L.M.: Contact parameter estimation for DEM simulation of iron ore pellet handling. Powder Technol. 248, 84-93 (2013)

[65] Coetzee, C.J.: Calibration of the discrete element method and the effect of particle shape. Powder Technol. 297, 50-70 (2016)

[66] Coetzee, C.J.: Review: Calibration of the discrete element method. Powder Technol. 310, 104-142 (2017)

[67] Elskamp, F., Hennig, M., Kruggel-Emden, H., Teipel, U.: A strategy to determine DEM parameters for spherical and non-spherical particles. Granul. Matter 19:46, (2017) 
[68] Zhu, H.P., Zhou, Z.Y., Yang, R.Y., Yu, A.B.: Discrete particle simulation of particulate systems: A review of major applications and findings. Chem. Eng. Sci. 63, 5728-5770 (2008)

[69] Munjiza, A., Latham, J.P., John, N.W.M.: 3D dynamics of discrete element systems comprising irregular discrete elements - integration solution for finite rotations in 3D. Int. J. Numer. Methods Eng. 56, 35-55 (2003)

[70] Kruggel-Emden, H., Simsek, E., Rickelt, S., Wirtz, S., Scherer, V.: Review and extension of normal force models for the Discrete Element Method. Powder Technol. 171, 157173 (2007)

[71] Kruggel-Emden, H., Wirtz, S., Scherer, V.: A study on tangential force laws applicable to the discrete element method (DEM) for materials with viscoelastic or plastic behavior. Chem. Eng. Sci. 63, 1523-1541 (2008)

[72] Iveson, S.M., Litster, J.D., Hapgood, K., Ennis, B.J.: Nucleation, growth and breakage phenomena in agitated wet granulation processes : a review. Powder Technol. 117, 339 (2001)

[73] Butt, H.-J., Kappl, M.: Surface and Interfacial Forces. Wiley-VCH Verlag, Weinheim (2010)

[74] Young, T.: An Essay on the Cohesion of Fluids. Philos. Trans. R. Soc. 95, 65-87 (1805)

[75] Mio, H., Shimosaka, A., Shirakawa, Y., Hidaka, J.: Cell optimization for fast contact detection in the discrete element method algorithm. Adv. Powder Technol. 18, 441-453 (2007)

[76] Kačianauskas, R., Maknickas, A., Kačeniauskas, A., Markauskas, D., Balevičius, R.: Parallel discrete element simulation of poly-dispersed granular material. Adv. Eng. Softw. 41, 52-63 (2010)

[77] Natsui, S., Sawada, A., Terui, K., Kashihara, Y., Kikuchi, T., Suzuki, R.O.: DEM-SPH study of molten slag trickle flow in coke bed. Chem. Eng. Sci. 175, 25-39 (2018) 